\title{
Design, Synthesis and Anticancer Activity of $N^{3}, N^{11}$-Bis(2-hydroxyethyl)- 14-aryl-14H-dibenzo[ $a, j]$ xanthenes-3,11-dicarboxamide
}

\author{
Yongbin Song, ${ }^{a}$ Yihui Yang, ${ }^{b}$ Jun You, ${ }^{a}$ Bo Liu,,${ }^{* a}$ Lijun Wu, ${ }^{b}$ Yunlong Hou, ${ }^{b}$ Wenji Wang, ${ }^{b}$ and \\ Jiuxin $\mathrm{Zhu}^{b}$ \\ ${ }^{a}$ Key Laboratory of Green Chemical Technology of College of Heilongjiang Province, School of Chemical and \\ Environmental Engineering, Harbin University of Science and Technology; Harbin 150040, China: and ${ }^{b}$ College of \\ Pharmacy, Harbin Medical University; Harbin 150081, China. \\ Received August 16, 2012; accepted October 30, 2012; advance publication released online November 12, 2012
}

A series of novel $N^{3}, N^{11}$-bis(2-hydroxyethyl)-14-aryl-14H-dibenzo[ $[a, j]$ xanthenes-3,11-dicarboxamide, three $\quad N^{3}, N^{11}$-bis(2-hydroxyethyl)-14-aryl-14H-dibenzo $[a, j]$ xanthene-3,11-dimethanamine derivatives and their intermediates 14-aryl-14H-dibenzo $[a, j]$ xanthenes-3,11-dicarboxylic acid, were synthesized, and the structures of which were characterized by ${ }^{1} \mathrm{H}-\mathrm{NMR},{ }^{13} \mathrm{C}-\mathrm{NMR}$, high resolution (HR)-MS, and IR spectra. The antitumor activities of these molecules were evaluated on five cancer cell lines. The results of in vitro assay against human hepatocellular carcinoma cell lines (SK-HEP-1 and HepG2 and SMMC-7721 cells), acute promyelocytic leukemia NB4 cells and uterine cervix cancer HeLa cells, show several compounds to be endowed with cytotoxicity in micromolar to submicromolar range. The carboxamide derivatives $6 \mathrm{c}$ and 6e exhibitted good inhibition on NB4 cancer cells, and the $\mathrm{IC}_{50}$ values of which were $0.82 \mu \mathrm{M}$ and $0.96 \mu \mathrm{M}$, respectively, much lower than $5.01 \mu \mathrm{M}$ of the positive control $\mathrm{As}_{2} \mathrm{O}_{3}$. Flow cytometric analysis results revealed that compounds $6 \mathrm{e}$ and $6 \mathrm{f}$ may induce tumor cell apoptosis.

Key words dibenzo $[a, j]$ xanthene; synthesis; antitumor activity; cell apoptosis; NMR

The synthesis of xanthenes, especially benzoxanthenes has received much attention in recent years due to their wide range of biological and pharmacological activities such as antiviral, ${ }^{1)}$ antibacterial, ${ }^{2)}$ and anti-inflammatory ${ }^{3)}$ activities as well as the activities in photodynamic therapy ${ }^{4}$ and the activities as antagonists for the paralyzing action of zoxazolamine. ${ }^{5)}$ Furthermore, they can be used as dyes, $\left.{ }^{6}\right) \mathrm{pH}$-sensitive fluorescent materials for visualization of biomolecules ${ }^{7)}$ and utilized in laser technologies. ${ }^{8)}$

Anthracenes, especially anthraquinones, are the basic framework of anthracycline antitumor agents, which contain many clinical drugs such as bisantrene, doxorubicin, daunorubicin, epirubicin, zorubicin, and aclarubicin. Mitoxantrone (Fig. 1) is a significant synthesized antineoplastic agent, which owns strong clinical activity on patients with breast cancer, acute leukemia and lymphoma. In addition, it exhibits antiviral, antibacterial, antiprotozoal, immunomodulating, and antineoplastic properties. ${ }^{9)}$ Therefore, it is significant to investigate the analogues of mitoxantrone which maintain its biological activity.

The findings of research indicate that the compounds of 14-aryl-14H-dibenzo[ $[a, j]$ xanthenes (Fig. 1) exhibit in vitro<smiles>O=C1c2c(O)ccc(O)c2C(=O)c2c(NCCNCCO)ccc(NCCNCCO)c21</smiles>

cytotoxicity against tumor cell lines, ${ }^{10}$ encouraging us to modify the structures of these derivatives. So far the synthesis of dibenzo[a,j]xanthene has been mostly focused on modification of 14-position of the heterocyclic ring, with other positions seldom changed. In order to study the relationship between the structures of mitoxantrone analogues and their biological activities, as well as to obtain compounds endowed with antiproliferative activity, we have developed a new series of 14-aryl-14H-dibenzo[ $a, j]$ xanthene-3,11-dicarboxamide compounds bearing a 2-hydroxyethyl group in the nitrogen atom (6a-i, Fig. 2). In addition, three $N^{3}, N^{11}$-bis(2-hydroxyethyl)-14aryl-14H-dibenzo[ $a, j]$ xanthene-3,11-dimethanamine derivatives (9a, 9e, 9f, Fig. 3) and ten intermediates containing dicarboxylic acid groups (5a-j, Fig. 2) were prepared. In this paper, we reported on the synthesis of target derivatives and theirs intermediates, and tested cytotoxicity on five cancer cell lines.

\section{Results and Discussion}

Chemistry The synthetic route for the new dicarboxamide derivatives $\mathbf{6 a}-\mathbf{i}$ is outlined in Chart 1. Compounds 1-4, which were known compounds, were the materials and

$$
\text { Mitoxantrone 14-aryl-14H-dibenzo[a,j]xanthene } \mathrm{R}=\text { aryl }
$$

Fig. 1. Structures of Mitoxantrone and 14-Aryl-14H-dibenzo[a,j]xanthenes 


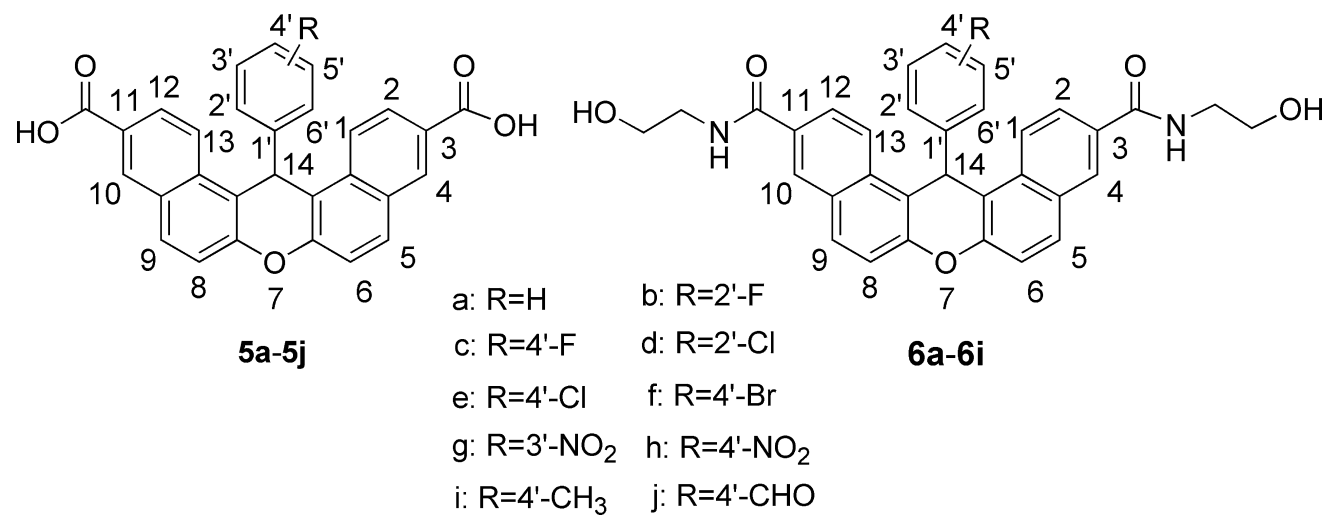

Fig. 2. Structures of $\mathbf{5 a}-\mathbf{j}$ and $\mathbf{6} \mathbf{a}-\mathbf{i}$

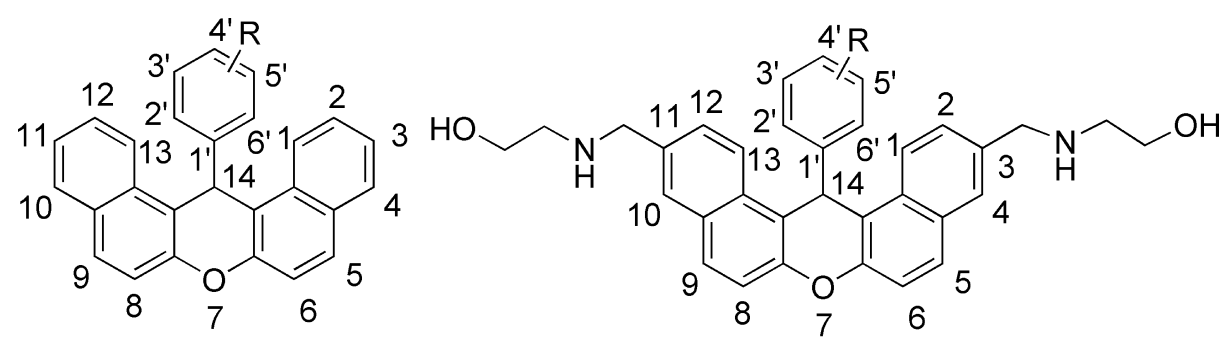

$7 a, 7 e$ and $7 f$

a: $\mathrm{R}=\mathrm{H}$

9a,9e and $9 f$

e: $R=4^{\prime}-C l \quad f: R=4^{\prime}-B r$

Fig. 3. Structures of 7a, 7e, $7 \mathbf{f}$ and $9 a, 9 e, 9 f$<smiles>N#Cc1ccc2cc(O)ccc2c1</smiles>

1

2<smiles>[R]c1cccc(C2c3c(ccc4cc(C(=O)O)ccc34)Oc3ccc4cc(C(=O)Cc5cc(C=O)ccc5[R])ccc4c32)c1</smiles>

4

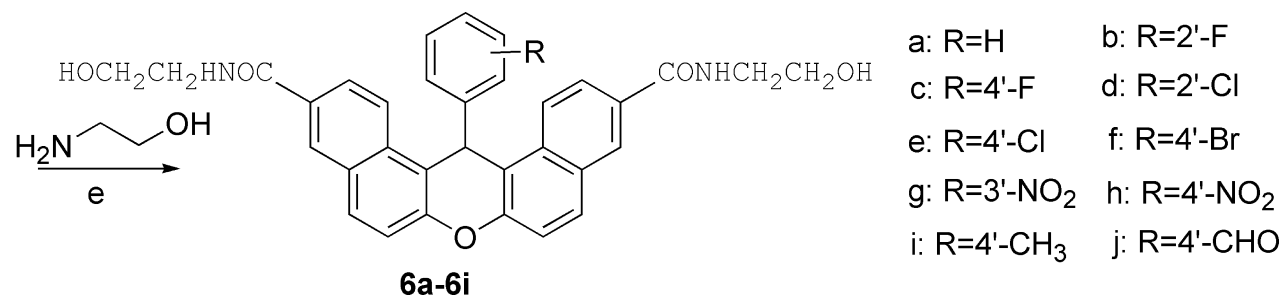

Reagents and conditions: (a) (i) $\mathrm{Br}_{2}$, $\mathrm{AcOH}$, (ii) $\mathrm{Sn}, \mathrm{AcOH}, 3 \mathrm{~h}$, reflux; (b) $\mathrm{CuCN}, \mathrm{DMF}, 4 \mathrm{~h}, 160^{\circ} \mathrm{C}$; (c) $\mathrm{HCl}_{2} \mathrm{H}_{2} \mathrm{O}, \mathrm{reflux} 8 \mathrm{~h}$; (d) $\mathrm{H}_{2} \mathrm{SO}$, $\mathrm{AcOH}$, 0.5-2 h; (e) (i) $\mathrm{SOCl}{ }_{2}$, $\mathrm{DMF}, \mathrm{CHCl}_{3}, 4 \mathrm{~h}, 67^{\circ} \mathrm{C}$; (ii) $\mathrm{CHCl}_{3}, 3 \mathrm{~h}, \mathrm{rt}$.

Chart 1

intermediates for the synthesis of dicarboxamide derivatives (6a-i). Compounds $\mathbf{2}$ and $\mathbf{3}$ were prepared through published methodology, ${ }^{11,12)}$ respectively. Compound $\mathbf{3}$ was refluxed for $8 \mathrm{~h}$ in the presence of $10 \%$ hydrochloric acid, the formed precipitate was filtered and washed with water and EtOAc successively, and then dried under vacuum to afford the 6-hydroxy-2-naphthalenecarboxylic acid compound 4 (88-90\% yield) (Chart 1).
The synthesis of key intermediate dicarboxylic acids $(\mathbf{5 a}-\mathbf{j})$ is also outlined in Chart $1 . \mathbf{5} \mathbf{a}-\mathbf{j}$ were prepared through the one-pot condensation of aldehydes with compound $\mathbf{4}$ in the present of concentrated sulfuric acid in acetic acid and good yields (84-92\%) were obtained.

Compounds $\mathbf{5 a}-\mathbf{i}$ were firstly converted into the corresponding acyl chlorides, and then turned into dicarboxamides 6a-i (84-92\% yield) (Chart 1), by adding excessive 
<smiles>[R][X]c1cccc(C2c3c(ccc4cc(C(=O)O)ccc34)Oc3ccc4cc(C(=O)O)ccc4c32)c1</smiles>

$5 a, 5 e$ and $5 f$<smiles>[R][X]c1ccc(C2c3c(ccc4cc(CO)ccc34)Oc3ccc4cc(CO)ccc4c32)cc1</smiles>

$8 a, 8 e$ and $8 f$<smiles>[R]c1cccc(C2c3c(ccc4cc(CNCCO)ccc34)Oc3ccc4cc(CNCCO)ccc4c32)c1</smiles>

9a, 9e and $9 f$

Reagents and conditions: (f) (i) $\mathrm{SOCl}_{2}, \mathrm{DMF}, \mathrm{CHCl}_{3}, 4 \mathrm{~h}, 67^{\circ} \mathrm{C}$, (ii) $\mathrm{LiAlH}_{4}, \mathrm{THF}, 15 \mathrm{~min}, 0^{\circ} \mathrm{C}$; (g) (i) $\mathrm{SOCl}_{2}, \mathrm{DMF} \mathrm{CHCl}_{3}, 0.5 \mathrm{~h}, \mathrm{r}_{\text {.t. }}$; (ii) 2 -aminoethanol, $\mathrm{CHCl}$, $6 \mathrm{~h}$, r.t. Chart 2

2-aminoethanol. But the conversion of compound $\mathbf{5 j}$ into the corresponding dicarboxamide $\mathbf{6 j}$ was unsuccessful, and this may be due to the formyl group of $\mathbf{5 j}$. It is easy to purify the compounds type $\mathbf{5}$ and $\mathbf{6}$, which have small solubilities in the corresponding solvents, and the dopants are soluble.

Known compounds $\mathbf{7 a}, \mathbf{7 e}$ and $\mathbf{7 f}$ (Fig. 3) were prepared through published methodology. ${ }^{13)}$ The synthetic route for the new dimethanamine derivatives 9a, 9e and 9f (Fig. 3) is outlined in Chart 2. The conversions of compounds, 6a, 6e and $\mathbf{6 f}$ into the corresponding dimethanamines produce very low yields using lithium aluminum hydride as reducing agent in tetrahydrofuran (THF), possibly owing to side reactions and the reduction of the halogen atoms in these compounds. Compounds 5a and $\mathbf{5 e}$ can give $\mathbf{8 a}$ and $\mathbf{8 e}$, respectively, using lithium aluminum hydride as reducing agent, but $\mathbf{5 f}$ only gives $\mathbf{8 a}$, not $\mathbf{8 f}$, owing to the reduction of the bromine atom into hydrogen atom. $\mathrm{LiAlH}_{4}$ can convert acid chloride of dicarboxylic acid $\mathbf{5}$ into compounds $\mathbf{8}$ quickly, and the bromine atom of $\mathbf{5 f}$ can be retained in the dimethanol derivative $\mathbf{8 f}$. Compounds $\mathbf{8}$ were treated with thionyl chloride to give corresponding dichlorinated compounds, which were not separated and reacted with 2-aminoethanol in $\mathrm{CHCl}_{3}$ to afford derivatives $9 \mathbf{a}, \mathbf{9 e}$ and $\mathbf{9 f}$.

All the structures of the synthetic intermediates $(\mathbf{5 a}-\mathbf{j})$, the derivatives $(\mathbf{6 a}-\mathbf{i}, \mathbf{8 a}, \mathbf{8 e}, \mathbf{8 f}, 9 \mathbf{9}, 9 \mathbf{e}, 9 \mathbf{9})$ were confirmed by ${ }^{1} \mathrm{H}-\mathrm{NMR},{ }^{13} \mathrm{C}-\mathrm{NMR}$, high resolution (HR)-MS, IR spectroscopic data which allowed the correct identification and determined the purity of the compounds.

Antiproliferation Assay The series of compounds 5a-j, $\mathbf{6 a}-\mathbf{i}, 9 \mathbf{9}, 9 \mathrm{e}$ and $9 \mathrm{f}$ were tested for cytotoxicity against a panel of five cancer cell lines of different types of human tumors, which contained human hepatoma cell (SK-HEP-1, HepG2, SMMC-7721), acute promyelocytic leukemia cell (NB4), and uterine cervix cancer cell (HeLa). The corresponding $\mathrm{IC}_{50}$ values of all compounds are summarized in Table 1, and arsenic trioxide $\left(\mathrm{As}_{2} \mathrm{O}_{3}\right)$ tested in parallel was used as cytotoxic positive control. In addition, dibenzo $[a, j]$ xanthene derivatives (7e, 7e, 7f), which do not possess functional groups on 3- and 11-positions, were used as negative standards, and they don't show antitumor activity to five cancer cell lines $\left(\mathrm{IC}_{50}>50 \mu \mathrm{M}\right)$. As evidenced by the cytotoxicity data, the carboxamide derivatives $\mathbf{6 a}-\mathbf{i}$ show better inhibitory activity than carboxylic acid derivatives $\mathbf{5 a}-\mathbf{j}$ in most cases. Meanwhile, compounds with a substituted benzene ring at the position 14 of the molecule $(\mathbf{6} \mathbf{c}-\mathbf{i}, \mathbf{5 b}-\mathbf{i})$ appear to be more toxic than those with an unsubstituted benzene ring (6a, 5a); moreover, a comparison of the substitution on the benzene ring suggests that para position-halogen-substituted carboxamide derivatives (e.g., 6c) exhibit stronger inhibitory activity for the cancer cells than those of ortho position-halogen-substituted ones (e.g., $\mathbf{6 b}$, Table 1).

Compounds $\mathbf{6 c}, \mathbf{6 e}, \mathbf{6} \mathbf{f}$ and $\mathbf{6 h}$ are proved to be potent cytotoxic agents towards leukemia cell line NB4. The carboxamide derivative $\mathbf{6 c}$ demonstrates the best inhibition to NB4 cancer cells at a concentration of $0.82 \mu \mathrm{M}$, lower than $5.01 \mu \mathrm{M}$ of $\mathrm{As}_{2} \mathrm{O}_{3}$. And the mean $\mathrm{IC}_{50}$ values of $\mathbf{6 e}$, $\mathbf{6} \mathbf{f}$ and $\mathbf{6 h}$ are 0.96 , 2.06 and $2.38 \mu \mathrm{M}$, respectively, which are also lower than that of arsenic trioxide (Table 1). Compound $6 \mathbf{i}$ inhibits the growth of NB4 cells at $6.15 \mu \mathrm{M}$, which is near the concentration of $\mathrm{As}_{2} \mathrm{O}_{3}$. In addition, carboxylic acid compounds $\mathbf{5 b}-\mathbf{i}$ exhibit a moderate cytotoxicity to NB4 cell line; in contrast, the ana$\operatorname{logs}_{5} \mathbf{5 a}$ and $\mathbf{5} \mathbf{j}$ are inactive (Table 1 ). the mean $\mathrm{IC}_{50}$ values of 9a, 9e and 9f are 3.08, 4.12 and $4.01 \mu \mathrm{M}$, respectively, which are also lower than that of arsenic trioxide (Table 1).

The derivatives $\mathbf{6 c}$ and $\mathbf{6 d}$ exhibit respective $\mathrm{IC}_{50}$ values of 5.72 and $5.26 \mu \mathrm{M}$ to human hepatoma cell line (HepG2), which are lower than the positive control. The $\mathrm{IC}_{50}$ value of compound 6e to HepG2 cell line is $6.41 \mu \mathrm{M}$, which is comparable to that of the positive control $\mathrm{As}_{2} \mathrm{O}_{3}$. Compounds $6 f$ and $6 \mathrm{~g}$ exhibit moderate $\mathrm{IC}_{50}$ values of 11.56 and $16.07 \mu \mathrm{M}$ to HepG2 cell line, respectively, and $\mathbf{6 i}$ shows a weak $\mathrm{IC}_{50}$ value of $30.47 \mu \mathrm{M}$ (Table 1). The derivatives 9a, 9e, and 9f exhibit $\mathrm{IC}_{50}$ values of $13.24,14.49$, and $16.79 \mu \mathrm{M}$ to HepG2 cell line, respectively (Table 1$)$.

Compounds 6e and $\mathbf{6 f}$ exhibit a good inhibitory effect on human hepatoma cell line (SK-HEP-1), and the $\mathrm{IC}_{50}$ values are 6.46 and $6.41 \mu \mathrm{M}$, respectively, which are comparable to that of $\mathrm{As}_{2} \mathrm{O}_{3}(6.02 \mu \mathrm{M}$, Table 1). Compound $\mathbf{6 e}$ is also proved 
Table 1. $\mathrm{IC}_{50}$ Values $(\mu \mathrm{M})$ for Antiproliferative Activity of the Synthesized Compounds

\begin{tabular}{|c|c|c|c|c|c|}
\hline \multirow{2}{*}{ Compound } & \multicolumn{5}{|c|}{$\mathrm{IC}_{50}(\mu \mathrm{M})$ for different cell lines ${ }^{a)}$} \\
\hline & HepG2 & SK-HEP-1 & SMMC-7721 & NB4 & $\mathrm{HeLa}$ \\
\hline $5 a$ & $>50$ & $>50$ & $>50$ & $>50$ & $>50$ \\
\hline $5 \mathbf{b}$ & $>50$ & $>50$ & $>50$ & 22. & $21.17 \pm 0.34$ \\
\hline $5 c$ & $>50$ & $>50$ & $>50$ & $18.21 \pm 1.43$ & $>50$ \\
\hline $5 d$ & $>50$ & $>50$ & $>50$ & $30.35 \pm 1.17$ & $22.13 \pm 1.02$ \\
\hline $5 e$ & $>50$ & $>50$ & $>50$ & $23.24 \pm 1.39$ & $>50$ \\
\hline $5 f$ & $>50$ & $>50$ & $>50$ & $17.33 \pm 0.14$ & $36.39 \pm 1.48$ \\
\hline $5 g$ & $>50$ & $>50$ & $>50$ & $37.27 \pm 0.74$ & $>50$ \\
\hline $5 \mathrm{~h}$ & $>50$ & $>50$ & $>50$ & $44.41 \pm 1.02$ & $>50$ \\
\hline $5 i$ & $>50$ & $>50$ & $>50$ & $47.64 \pm 1.38$ & $>50$ \\
\hline $5 \mathbf{j}$ & $>50$ & $>50$ & $>50$ & $>50$ & $>50$ \\
\hline $6 a$ & $>50$ & $>50$ & $>50$ & $>50$ & $>50$ \\
\hline $6 b$ & $>50$ & $>50$ & $>50$ & $>50$ & $>50$ \\
\hline $6 c$ & $5.72 \pm 0.54$ & $9.81 \pm 0.38$ & $12.50 \pm 1.16$ & $0.82 \pm 0.042$ & $27.94 \pm 2.58$ \\
\hline 6d & $5.26 \pm 0.96$ & $9.21 \pm 1.84$ & $>50$ & $>50$ & $21.51 \pm 2.05$ \\
\hline $6 e$ & $6.41 \pm 0.032$ & $6.46 \pm 0.50$ & $9.92 \pm 1.06$ & $0.96 \pm 0.051$ & $17.72 \pm 1.83$ \\
\hline $6 f$ & $11.56 \pm 0.69$ & $6.41 \pm 0.52$ & $18.71 \pm 0.65$ & $2.06 \pm 0.21$ & $13.18 \pm 1.79$ \\
\hline $6 \mathrm{~g}$ & $16.07 \pm 0.42$ & $>50$ & $>50$ & $>50$ & $>50$ \\
\hline $6 \mathrm{~h}$ & $>50$ & $19.27 \pm 1.35$ & $>50$ & $2.38 \pm 0.27$ & $>50$ \\
\hline $6 i$ & $30.47 \pm 1.98$ & $13.98 \pm 1.01$ & $20.11 \pm 0.24$ & $6.15 \pm 0.94$ & $20.76 \pm 1.95$ \\
\hline $9 a$ & $13.24 \pm 0.52$ & $7.35 \pm 0.29$ & $16.68 \pm 0.61$ & $3.08 \pm 0.25$ & $14.27 \pm 1.63$ \\
\hline $9 e$ & $14.49 \pm 0.35$ & $7.31 \pm 0.72$ & $17.25 \pm 0.67$ & $4.12 \pm 0.32$ & $15.18 \pm 1.79$ \\
\hline 9f & $16.79 \pm 0.41$ & $8.44 \pm 0.34$ & $16.35 \pm 0.68$ & $4.01 \pm 0.41$ & $16.35 \pm 2.13$ \\
\hline $7 \mathbf{a}, 7 \mathbf{e}, 7 \mathbf{f}$ & $>50$ & $>50$ & $>50$ & $>50$ & $>50$ \\
\hline $\mathrm{As}_{2} \mathrm{O}_{3}$ & $6.23 \pm 0.34$ & $6.02 \pm 0.38$ & $9.13 \pm 0.82$ & $5.01 \pm 0.071$ & $10.92 \pm 0.22$ \\
\hline
\end{tabular}

a) Values are means \pm standard deviation from three consecutive experiments.

to be more active in inhibition of human hepatoma cell (SMMC-7721) than its analogues. Compound 6 f shows a better inhibitory activity than its derivatives to uterine cervix cancer HeLa cells (Table 1).

It can be found that halogen substitution at C-4' (para) position on the 14-phenyl group of dicarboxamide derivatives $\mathbf{6 a}-\mathbf{i}$ is critical for improving the antitumor activity to five cell lines, especially to eukemia cell line NB4. The ability sequence of the halogen atoms in compounds $\mathbf{6 c}, \mathbf{6 e}$ and $\mathbf{6}$ f to improve the antitumor activity is $\mathrm{F}>\mathrm{Cl}>\mathrm{Br}$, which maybe due to the atomic size difference or the capability of forming hydrogen bonds with the receptor. Halogen substitution at C-2' (ortho) position on the 14-phenyl group shows little effect on antitumor activity, thus we can deduce that the steric hindrance can block the conjunction between the halogen atom and receptor. Derivatives 9a, 9e and 9f show a comparative antitumor activity with that of $\mathbf{6} \mathbf{f}$ to five cell lines (Table 1). An unsubstituted benzene ring at the position 14 of the molecule (9a) appears to be more toxic than those with a substituted benzene ring $(\mathbf{9 e}, \mathbf{9 f})$, and the antitumor activities of derivatives 9a, 9e and $9 \mathbf{f}$ do not show a similar tendency toward halogen substitution with that of compounds $\mathbf{6 a}-\mathbf{a}$.

Several compounds show a high level of tumor cells selectivity. For instance, Compound 6d shows anti-proliferative activity on human hepatoma SK-HEP-1, HepG2 and uterine cervix cancer HeLa cells, but it does not exhibit cytotoxic activity on human hepatoma SMMC-7721 and acute promyelocytic leukemia NB4 cells, the $\mathrm{IC}_{50}$ values of which exceed $50 \mu \mathrm{M}$. Compound $\mathbf{6 g}$ shows only anti-proliferative activity to cell line HepG2, and doesn't exhibit inhibitory activity to other tumor cell lines (Table 1).

Apoptosis Assay On the basis of the antiproliferative effect study, compounds $\mathbf{6 e}$ and $\mathbf{6 f}$ were selected to perform a biparametric cytofluorimetric analysis using annexin $\mathrm{V}$ and PI double-staining as shown in Fig. 4, in order to elucidate whether the compound-induced cell death involved apoptosis or necrosis. The results indicate that the experimental compounds could induce the apoptosis of activated HeLa cells. It has been found that compounds $\mathbf{6 e}$ and $\mathbf{6 f}$ induce more apoptotic cells than necrotic cells, and this phenomenon is even more pronounced at a lower concentration $(12.5 \mu \mathrm{M})$ than at a higher concentration $(25 \mu \mathrm{M})$.

\section{Conclusion}

In conclusion, a series of $N^{3}, N^{11}$-bis(2-hydroxyethyl)-14aryl-14H-dibenzo[ $a, j]$ xanthenes-3,11-dicarboxamide derivatives, three $N^{3}, N^{11}$-bis(2-hydroxyethyl)-14-aryl-14H-dibenzo$[a, j]$ xanthene-3,11-dimethanamine derivatives and their intermediate can be synthesized in acceptable overall yields. The results of in vitro antitumor activity experiments reveal that the series of compounds exhibit pronounced inhibitory activities toward a wide range of human tumor cell lines, and some of them show a high level of tumor cells selectivity. The carboxamide derivatives $\mathbf{6 c}$ and $\mathbf{6 e}$ demonstrate good inhibition effects on NB4 cancer cells, and the best activities appear at concentrations of $0.82 \mu \mathrm{M}$ and $0.96 \mu \mathrm{M}$, respectively, much lower than $5.01 \mu \mathrm{M}$ of the positive control $\mathrm{As}_{2} \mathrm{O}_{3}$, which is believed to be a potential antitumor agent with a potent inhibitory activity in tumor growth. Apoptosis assay results by the flow cytometry analysis show that compounds $\mathbf{6 e}$ and $\mathbf{6 f}$ are potential antitumor agents.

\section{Experimental}

Chemistry All the solvents were of analytic grade. Silica 

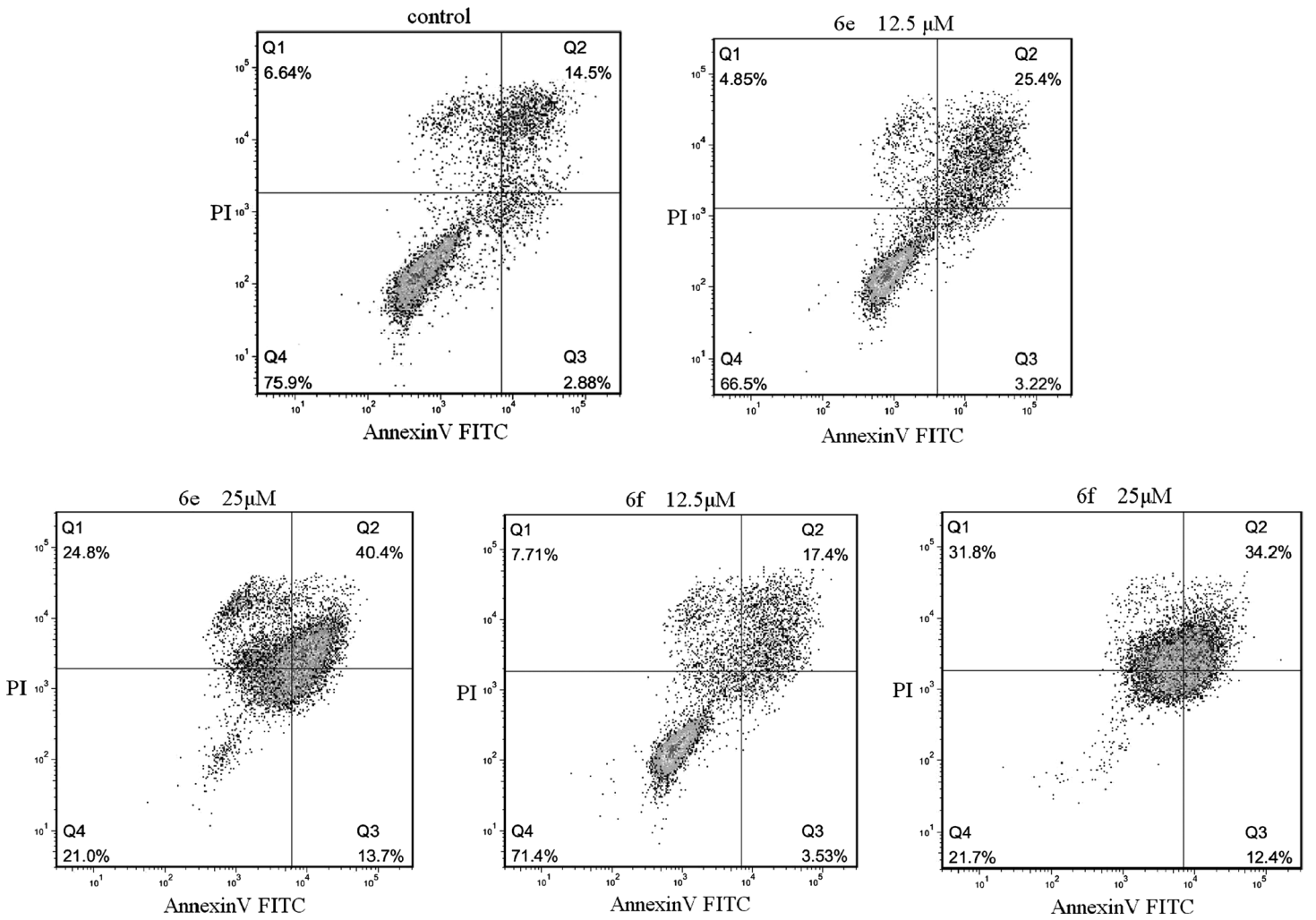

Fig. 4. The Apoptosis Induction Effect of $\mathbf{6 e}$ and $\mathbf{6 f}$ on HeLa Cells, HeLa Cells Were Cultured with Agents for $24 \mathrm{~h}$

Cells were stained by Annexin V-FITC/PI and apoptosis was analyzed by flow cytometry.

GF254 plates were purchased from Qingdao Haiyang Chemical Co., Ltd., China. The melting points of the compounds were taken on an X-6 melting piont apparatus and are uncorrected. IR spectra were obtained on an Avatar 370 FT-IR spectrometer. ${ }^{1} \mathrm{H}$ - and ${ }^{13} \mathrm{C}$-NMR spectra were recorded at 300 and $75 \mathrm{MHz}$, respectively in DMSO- $d_{6}$, using a Bruker Avance 300 spectrometer (tetramethylsilane (TMS) as an internal standard). HR-MS were measured on a Waters LCT Premier XE benchtop orthogonal acceleration time-of-flight mass spectrometer.

General Procedure for the Preparation of 14-Aryl-14H-Dibenzo[ $a, j]$ xanthene-3,11-dicarboxylic Acid (5a-j) A mixture of 6-hydroxy-2-naphthalenecarboxylic acid $43.76 \mathrm{~g}(20 \mathrm{mmol})$, appropriate arylaldehyde $(10.5 \mathrm{mmol})$, glacial acetic acid $(20 \mathrm{~mL})$ and concentrated sulfuric acid $(1 \mathrm{~mL})$ was stirred at room temperature for $10 \mathrm{~min}$, and then heated to reflux for $0.5-2 \mathrm{~h}$. TLC was used to monitor the reactions. After the reaction had been completed, the system was cooled to room temperature, the formed precipitate was filtered and washed with acetic acid and water successively, then dried under vacuum to afford the compounds $\mathbf{5 a}-\mathbf{j}$.

14-Phenyl-14H-dibenzo[ $a, j]$ xanthene-3,11-dicarboxylic Acid (5a): White solid. Yield 82.3\%. mp $>300{ }^{\circ} \mathrm{C} .{ }^{1} \mathrm{H}-\mathrm{NMR}$ $\left(\mathrm{DMSO}_{6}, 300 \mathrm{MHz}\right) \delta: 8.74(\mathrm{~d}, J=9.0 \mathrm{~Hz}, 2 \mathrm{H}, 1,13-\mathrm{H})$, $8.60(\mathrm{~d}, J=1.4 \mathrm{~Hz}, 2 \mathrm{H}, 4,10-\mathrm{H}), 8.14(\mathrm{~d}, J=8.9 \mathrm{~Hz}, 2 \mathrm{H}, 5,9-\mathrm{H})$, $8.06(\mathrm{dd}, J=9.0,1.5 \mathrm{~Hz}, 2 \mathrm{H}, 2,12-\mathrm{H}), 7.64(\mathrm{~d}, J=8.9 \mathrm{~Hz}, 2 \mathrm{H}$, 6, 8-H), 7.60 (d, J=7.3 Hz, 2H, 2', 6'-H), 7.15(t, J=7.4 Hz, $\left.2 \mathrm{H}, 3^{\prime}, 5^{\prime}-\mathrm{H}\right), 6.98$ (t, J=7.3 Hz, 1H, 4'-H), 6.76(s, 1H, 14-H). ${ }^{13} \mathrm{C}-\mathrm{NMR}$ (DMSO- $\left.d_{6}, 75 \mathrm{MHz}\right) \delta: 167.6,149.9,145.5,133.7$, $131.6,131.2,130.5,129.0,128.3,127.1,127.0,126.7,124.3$, 119.1, 118.0, 36.9. IR (KBr) v: 1686, 1620, 1465, 1396, 1300, $1242 \mathrm{~cm}^{-1}$. HR-MS (electrospray ionization (ESI)) Calcd for
$\mathrm{C}_{29} \mathrm{H}_{17} \mathrm{O}_{5}[\mathrm{M}-\mathrm{H}]^{+}$455.1076, Found 455.1071.

14-(2-Fluorophenyl)-14H-dibenzo[ $a, j]$ xanthene-3,11-dicarboxylic Acid (5b): White solid. Yield 83.4\%. mp $>300^{\circ} \mathrm{C}$. ${ }^{1} \mathrm{H}-\mathrm{NMR}$ (DMSO- $\left.d_{6}, 300 \mathrm{MHz}\right) \delta: 8.62(\mathrm{~d}, J=1.5 \mathrm{~Hz}, 2 \mathrm{H}, 4$, $10-\mathrm{H}), 8.50$ (d, $J=9.0 \mathrm{~Hz}, 2 \mathrm{H}, 1,13-\mathrm{H}), 8.18$ (d, $J=8.9 \mathrm{~Hz}, 2 \mathrm{H}$, $5,9-\mathrm{H}), 8.07(\mathrm{dd}, J=9.0,1.6 \mathrm{~Hz}, 2 \mathrm{H}, 2,12-\mathrm{H}), 7.65(\mathrm{~d}, J=$ $8.9 \mathrm{~Hz}, 2 \mathrm{H}, 6,8-\mathrm{H}), 7.59-7.62(\mathrm{~m}, 1 \mathrm{H}), 6.70-7.11(\mathrm{~m}, 3 \mathrm{H}), 6.88$ $(\mathrm{s}, 1 \mathrm{H}, 14-\mathrm{H}) .{ }^{13} \mathrm{C}-\mathrm{NMR}$ (DMSO- $\left.d_{6}, 75 \mathrm{MHz}\right) \delta$ : $167.7,158.9$ $\left({ }^{1} J_{\mathrm{CF}}=242.9 \mathrm{~Hz}\right), 150.0,133.6,131.8\left({ }^{3} J_{\mathrm{CF}}=7.0 \mathrm{~Hz}\right), 131.7,131.5$ $\left({ }^{2} J_{\mathrm{CF}}=24.4 \mathrm{~Hz}\right), 131.4,130.3,129.5\left({ }^{3} J_{\mathrm{CF}}=8.5 \mathrm{~Hz}\right), 127.3,126.9$, $125.6,123.1,118.9,116.2\left({ }^{2} J_{\mathrm{CF}}=22.5 \mathrm{~Hz}\right), 115.8,31.4$. IR $(\mathrm{KBr})$ $v: 1689,1621,1466,1398,1301,1242 \mathrm{~cm}^{-1}$. HR-MS (ESI) Calcd for $\mathrm{C}_{29} \mathrm{H}_{16} \mathrm{FO}_{5}[\mathrm{M}-\mathrm{H}]^{+}$463.0982, Found 463.0987.

14-(4-Fluorophenyl)-14H-dibenzo[a,j]xanthene-3,11-dicarboxylic Acid (5c): White solid. Yield 85.3\%. mp $>300^{\circ} \mathrm{C}$. ${ }^{1} \mathrm{H}-\mathrm{NMR}$ (DMSO- $\left.d_{6}, 300 \mathrm{MHz}\right) \delta: 8.77(\mathrm{~d}, J=9.0 \mathrm{~Hz}, 2 \mathrm{H}, 1$, $13-\mathrm{H}), 8.62$ (d, $J=1.4 \mathrm{~Hz}, 2 \mathrm{H}, 4,10-\mathrm{H}), 8.16(\mathrm{~d}, J=9.0 \mathrm{~Hz}, 2 \mathrm{H}$, 5, 9-H), 8.07 (dd, $J=9.0,1.4 \mathrm{~Hz}, 2 \mathrm{H}, 2,12-\mathrm{H}), 7.66(\mathrm{~d}, J=$ $9.0 \mathrm{~Hz}, 2 \mathrm{H}, 6,8-\mathrm{H}), 7.68-7.59\left(\mathrm{~m}, 2 \mathrm{H}, 2^{\prime}, 6^{\prime}-\mathrm{H}\right), 6.99(\mathrm{t}, J=$ $\left.8.8 \mathrm{~Hz}, 2 \mathrm{H}, 33^{\prime}, 5^{\prime}-\mathrm{H}\right), 6.82(\mathrm{~s}, 1 \mathrm{H}, 14-\mathrm{H}) .{ }^{13} \mathrm{C}-\mathrm{NMR}$ (DMSO$\left.d_{6}, 75 \mathrm{MHz}\right) \delta: 167.7,161.0\left({ }^{1} J_{\mathrm{CF}}=241.9 \mathrm{~Hz}\right), 149.9,141.7,133.5$, $131.7,131.3,130.5,130.1 \quad\left({ }^{3} J_{\mathrm{CF}}=8.0 \mathrm{~Hz}\right), 127.3,126.7,124.2$, $119.1,117.9,115.8\left({ }^{2} J_{\mathrm{CF}}=21.3 \mathrm{~Hz}\right), 36.0$. IR (KBr) $v: 2925,1691$, 1620, 1466, 1398, 1296, $1245 \mathrm{~cm}^{-1}$. HR-MS (ESI) Calcd for $\mathrm{C}_{29} \mathrm{H}_{16} \mathrm{FO}_{5}[\mathrm{M}-\mathrm{H}]^{+}$463.0982, Found 463.0989.

14-(2-Chlorophenyl)-14H-dibenzo[ $a, j]$ xanthene-3,11-dicarboxylic Acid (5d): White solid. Yield 84.1\%. mp $>300^{\circ} \mathrm{C}$. ${ }^{1} \mathrm{H}-\mathrm{NMR}$ (DMSO, 300 MHz) $\delta: 8.59$ (brs, 2H, 4, 10-H), 8.54 (d, $J=9.1 \mathrm{~Hz}, 2 \mathrm{H}, 1,13-\mathrm{H}), 8.10(\mathrm{~d}, J=9.0 \mathrm{~Hz}, 2 \mathrm{H}, 5,9-\mathrm{H})$, 8.04 (dd, $J=9.1,1.3 \mathrm{~Hz}, 2 \mathrm{H}, 2,12-\mathrm{H}), 7.54$ (d, $J=9.0 \mathrm{~Hz}$, $2 \mathrm{H}, 6,8-\mathrm{H}), 7.43$ (d, $\left.J=7.3 \mathrm{~Hz}, 1 \mathrm{H}, 6^{\prime}-\mathrm{H}\right), 7.30$ (d, $J=7.9 \mathrm{~Hz}$, $\left.1 \mathrm{H}, 3^{\prime}-\mathrm{H}\right), 7.12-6.96\left(\mathrm{~m}, 2 \mathrm{H}, 4^{\prime}, 5^{\prime}-\mathrm{H}\right), 6.66$ (s, 1H, 14-H). 
${ }^{13} \mathrm{C}-\mathrm{NMR}$ (DMSO, $\left.75 \mathrm{MHz}\right) \delta: 167.7,150.1,142.6,133.7,132.2$, $131.7,131.5,130.5,130.3,129.2,128.7,127.3,126.7,126.7$, 123.8, 119.1, 116.8, 35.1. IR (KBr) v: 1685, 1620, 1469, 1397 , $1303,1250 \mathrm{~cm}^{-1}$. HR-MS (ESI) Calcd for $\mathrm{C}_{29} \mathrm{H}_{16} \mathrm{ClO}_{5}[\mathrm{M}+\mathrm{H}]^{+}$ 479.0686, Found 479.0679.

14 -(4-Chlorophenyl)-14H-dibenzo[ $a, j]$ xanthene-3,11dicarboxylic Acid (5e): White solid. Yield 87.9\%. mp $>300^{\circ} \mathrm{C}$. ${ }^{1} \mathrm{H}-\mathrm{NMR}$ (DMSO- $\left.d_{6}, 300 \mathrm{MHz}\right) \delta: 8.75(\mathrm{~d}, J=9.0 \mathrm{~Hz}, 2 \mathrm{H}, 1$, $13-\mathrm{H}), 8.63(\mathrm{~d}, J=1.6 \mathrm{~Hz}, 2 \mathrm{H}, 4,10-\mathrm{H}), 8.17(\mathrm{~d}, J=8.9 \mathrm{~Hz}$, 2H, 5, 9-H), 8.07 (dd, J=9.0, 1.7 Hz, 2H, 2, 12-H), 7.66 (d, $J=8.9 \mathrm{~Hz}, 2 \mathrm{H}, 6,8-\mathrm{H}), 7.64$ (d, $\left.J=8.5 \mathrm{~Hz}, 2 \mathrm{H}, 2^{\prime}, 6^{\prime}-\mathrm{H}\right), 7.23$ (d, $\left.J=8.5 \mathrm{~Hz}, 2 \mathrm{H}, 3{ }^{\prime}, 55^{\prime}-\mathrm{H}\right), 6.82(\mathrm{~s}, 1 \mathrm{H}, 14-\mathrm{H}) .{ }^{13} \mathrm{C}-\mathrm{NMR}$ (DMSO- $\left.d_{6}, 75 \mathrm{MHz}\right) \delta: 167.7,149.9,144.5,133.5,131.6$, $131.6,131.4,130.5,130.1,129.0,127.4,126.8,124.2,119.1$, 117.6, 36.2. IR (KBr) v: 1694, 1621, 1466, 1397, 1245, 1157, $1135 \mathrm{~cm}^{-1}$. HR-MS (ESI) Calcd for $\mathrm{C}_{29} \mathrm{H}_{16} \mathrm{ClO}_{5}[\mathrm{M}+\mathrm{H}]^{+}$ 479.0686, Found 479.0689.

14 -(4-Bromophenyl)-14H-dibenzo[ $a, j]$ xanthene-3,11dicarboxylic Acid (5f): White solid. Yield 85.6\%. mp $>300^{\circ} \mathrm{C}$. ${ }^{1} \mathrm{H}-\mathrm{NMR}$ (DMSO- $\left.d_{6}, 300 \mathrm{MHz}\right) \delta: 8.76(\mathrm{~d}, J=8.8 \mathrm{~Hz}, 2 \mathrm{H}, 1$, 13-H), 8.63 (br s, 2H, 4, 10-H), 8.19 (d, $J=8.9 \mathrm{~Hz}, 2 \mathrm{H}, 5,9-\mathrm{H}$ ), 8.07 (brd, $J=8.8 \mathrm{~Hz}, 2 \mathrm{H}, 2,12-\mathrm{H}), 7.68$ (d, $J=8.9 \mathrm{~Hz}, 2 \mathrm{H}, 6$, $8-\mathrm{H}), 7.59$ (d, $\left.J=6.9 \mathrm{~Hz}, 2 \mathrm{H}, 2^{\prime}, 6^{\prime}-\mathrm{H}\right), 7.37$ (d, $J=6.5 \mathrm{~Hz}, 2 \mathrm{H}$, $\left.3^{\prime}, 5^{\prime}-\mathrm{H}\right), 6.82$ (s, 1H, 14-H). ${ }^{13} \mathrm{C}-\mathrm{NMR}$ (DMSO- $\left.d_{6}, 75 \mathrm{MHz}\right)$ $\delta: 167.7,149.9,144.9,133.5,132.0,131.7,131.4,130.5,130.5$, $127.3,126.8,124.2,120.2,119.1,117.5,36.3$. IR (KBr) v: 1703, 1621, 1467, 1400, 1300, $1252 \mathrm{~cm}^{-1}$. HR-MS (ESI) Calcd for $\mathrm{C}_{29} \mathrm{H}_{16} \mathrm{BrO}_{5}[\mathrm{M}-\mathrm{H}]^{+}$523.0181, Found 523.0188.

14-(3-Nitrophenyl)-14H-dibenzo[ $a, j]$ xanthene-3,11-dicarboxylic Acid (5g): White solid. Yield 84.3\%. mp $>300^{\circ} \mathrm{C}$. ${ }^{1} \mathrm{H}-\mathrm{NMR}\left(\mathrm{DMSO}-d_{6}, 300 \mathrm{MHz}\right) \delta: 8.84(\mathrm{~d}, J=9.0 \mathrm{~Hz}, 2 \mathrm{H}, 1$, $13-\mathrm{H}), 8.63$ (d, J=1.4 Hz, 2H, 4, 10-H), 8.52 (brs, 1H, 2'-H), 8.20 (d, $J=9.1 \mathrm{~Hz}, 2 \mathrm{H}, 5,9-\mathrm{H}), 8.10$ (brd, $\left.J=7.3 \mathrm{~Hz}, 1 \mathrm{H}, 6^{\prime}-\mathrm{H}\right)$, $8.08(\mathrm{dd}, J=9.0,1.5 \mathrm{~Hz}, 2 \mathrm{H}, 2,12-\mathrm{H}), 7.87(\mathrm{dd}, J=8.1,1.5 \mathrm{~Hz}$, $\left.1 \mathrm{H}, 4^{\prime}-\mathrm{H}\right), 7.70$ (d, J=9.0 Hz, 2H, 6, 8-H), 7.48 (t, $J=8.0 \mathrm{~Hz}$, $\left.1 \mathrm{H}, 5^{\prime}-\mathrm{H}\right), 7.03$ (s, $\left.1 \mathrm{H}, 14-\mathrm{H}\right) .{ }^{13} \mathrm{C}-\mathrm{NMR}$ (DMSO-d, $\left.75 \mathrm{MHz}\right)$ $\delta$ : 167.7, 150.1, 148.4, 147.4, 134.7, 133.5, 131.7, 130.7, 130.5, $127.5,126.9,124.1,122.5,122.3,119.1,117.1,117.1,36.3$. IR $(\mathrm{KBr}) v: 1686,1622,1534,1466,1397,1349,1246 \mathrm{~cm}^{-1}$. HR-MS (ESI) Calcd for $\mathrm{C}_{29} \mathrm{H}_{16} \mathrm{NO}_{7}[\mathrm{M}-\mathrm{H}]^{+}$490.0927, Found 490.0930 .

14-(4-Nitrophenyl)-14H-dibenzo[ $a, j]$ xanthene-3,11-dicarboxylic Acid (5h): White solid. Yield $82.3 \%$. mp $284-285^{\circ} \mathrm{C}$. ${ }^{1} \mathrm{H}-\mathrm{NMR}$ (DMSO- $\left.d_{6}, 300 \mathrm{MHz}\right) \delta: 8.78(\mathrm{~d}, J=8.9 \mathrm{~Hz}, 2 \mathrm{H}, 1$, 13-H), 8.64 (brs, 2H, 4, 10-H), 8.20 (d, $J=9.0 \mathrm{~Hz}, 2 \mathrm{H}, 5,9-\mathrm{H}$ ), 8.08 (brd, $J=8.9 \mathrm{~Hz}, 2 \mathrm{H}, 2,12-\mathrm{H}), 8.04$ (d, $J=8.4 \mathrm{~Hz}, 2 \mathrm{H}, 2^{\prime}$, $\left.6^{\prime}-\mathrm{H}\right), 7.92$ (d, $\left.J=8.3 \mathrm{~Hz}, 2 \mathrm{H}, 3^{\prime}, 5^{\prime}-\mathrm{H}\right), 7.70$ (d, $J=8.9 \mathrm{~Hz}, 2 \mathrm{H}$, 6, 8-H), 7.01 (s, 1H, 14-H). ${ }^{13} \mathrm{C}-\mathrm{NMR}$ (DMSO- $d_{6}, 75 \mathrm{MHz}$ ) $\delta: 167.7,152.5,150.0,146.4,133.5,131.7,131.7,130.5,129.5$, $127.4,126.9,124.4,124.1,119.1,116.8,36.7$. IR (KBr) v: 1703, 1621, 1522, 1467, 1400, 1345, $1251 \mathrm{~cm}^{-1}$. HR-MS (ESI) Calcd for $\mathrm{C}_{29} \mathrm{H}_{16} \mathrm{NO}_{7}[\mathrm{M}-\mathrm{H}]^{+}$490.0927, Found 490.0924.

14-(4-Methylphenyl)-14H-dibenzo[ $a, j]$ xanthene-3,11-dicarboxylic Acid (5i): White solid. Yield 84.9\%. mp $>300^{\circ} \mathrm{C}$. ${ }^{1} \mathrm{H}-\mathrm{NMR}$ (DMSO- $\left.d_{6}, 300 \mathrm{MHz}\right) \delta: 8.74(\mathrm{~d}, J=9.0 \mathrm{~Hz}, 2 \mathrm{H}, 1$, $13-\mathrm{H}), 8.61$ (s, 2H, 4, 10-H), 8.14 (d, J=9.0 Hz, 2H, 5, 9-H), $8.05(\mathrm{~d}, J=9.0 \mathrm{~Hz}, 2 \mathrm{H}, 2,12-\mathrm{H}), 7.64(\mathrm{~d}, J=8.9 \mathrm{~Hz}, 2 \mathrm{H}, 6$, $8-\mathrm{H}), 7.48$ (d, $\left.J=7.8 \mathrm{~Hz}, 2 \mathrm{H}, 2^{\prime}, 6^{\prime}-\mathrm{H}\right), 6.95$ (d, $J=7.8 \mathrm{~Hz}, 2 \mathrm{H}$, 3', 5'-H), $6.72(\mathrm{~s}, 1 \mathrm{H}, 14-\mathrm{H}), 2.04\left(\mathrm{~s}, 3 \mathrm{H}, \mathrm{CH}_{3}\right) .{ }^{13} \mathrm{C}-\mathrm{NMR}$ (DMSO- $\left.d_{6}, 75 \mathrm{MHz}\right) \delta: 167.8,149.8, \quad 142.7,136.1,133.6$, $131.6,131.1,130.5,129.5,128.2,127.2,126.6,124.4,119.0$,
118.1, 36.5; 20.9. IR (KBr) v: 1704, 1681, 1621, 1468, 1399, 1289, $1251 \mathrm{~cm}^{-1}$. HR-MS (ESI) Calcd for $\mathrm{C}_{30} \mathrm{H}_{19} \mathrm{O}_{5}[\mathrm{M}-\mathrm{H}]^{+}$ 459.1233, Found 459.1236.

14-(4-Formylphenyl)-14H-dibenzo[ $a, j]$ xanthene-3,11dicarboxylic Acid (5j): White solid. Yield 85.8\%. $\mathrm{mp}>300^{\circ} \mathrm{C}$. ${ }^{1} \mathrm{H}-\mathrm{NMR}$ (DMSO- $\left.d_{6}, 300 \mathrm{MHz}\right) \delta: 9.76$ (s, 1H, CHO), 8.78 (d, $J=9.1 \mathrm{~Hz}, 2 \mathrm{H}, 1,13-\mathrm{H}), 8.62$ (d, $J=1.7 \mathrm{~Hz}, 2 \mathrm{H}, 4,10-\mathrm{H})$, 8.19 (d, $J=9.1 \mathrm{~Hz}, 2 \mathrm{H}, 5,9-\mathrm{H}), 8.07$ (dd, $J=9.0,1.7 \mathrm{~Hz}, 2 \mathrm{H}$, 2, 12-H), 7.87 (d, $\left.J=8.2 \mathrm{~Hz}, 2 \mathrm{H}, 2^{\prime}, 6^{\prime}-\mathrm{H}\right), 7.71(\mathrm{~d}, J=8.2 \mathrm{~Hz}$, 2H, 3', 5'-H), 7.69 (d, J=8.9 Hz, 2H, 6, 8-H), 6.93 (s, 1H, 14-H). ${ }^{13} \mathrm{C}-\mathrm{NMR}$ (DMSO- $\left.d_{6}, 75 \mathrm{MHz}\right) \delta: 192.7,167.7,151.8$, $149.9,135.0,133.5,131.6,131.5,130.5,130.3,129.0,127.3$, 126.8, 124.1, 119.1, 117.1, 37.0. IR (KBr) v: 1708, 1692, 1621, 1468, 1399, 1299, $1251 \mathrm{~cm}^{-1}$. HR-MS (ESI) Calcd for $\mathrm{C}_{30} \mathrm{H}_{17} \mathrm{O}_{6}$ $[\mathrm{M}-\mathrm{H}]^{+}$473.1025, Found 473.1029.

General Procedure for the Preparation of $N^{3}, N^{11}$-Bis(2hyd roxyethyl)-14-aryl-14H-dibenzo $[a, j]$ xanthene-3,11dicarboxamide (6a-i) A $100 \mathrm{~mL}$, three-necked flask, equipped with a magnetic stir bar, a condenser, a thermometer and an addition funnel, was charged with $2 \mathrm{mmol}$ dibenzo[a,j]xanthenes dicarboxylic acid 5, $20 \mathrm{~mL}$ of $\mathrm{CHCl}_{3}$ and 1 drop of dimethylformamide. Afterwards a solution of $2.2 \mathrm{~mL}$ ( $30 \mathrm{mmol})$ thionyl chloride in $\mathrm{CHCl}_{3}(8 \mathrm{~mL})$ was added, and the mixture was heated to reflux at $67^{\circ} \mathrm{C}$ for $4 \mathrm{~h}$. After the initial suspension turned into a yellow solution, thionyl chloride was removed by evacuation under reduced pressure to give the

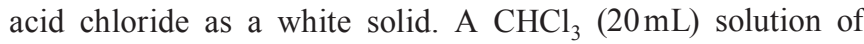
the acid chloride was then added to the solution of 2-aminoethanol $(488 \mathrm{mg}, 8 \mathrm{mmol})$ in $\mathrm{CHCl}_{3}(25 \mathrm{~mL})$. The mixture was stirred for $3 \mathrm{~h}$ at room temperature. A white solid formed was filtered and washed with $\mathrm{CHCl}_{3}$ and water, successively, then dried under vacuum to afford the compounds $\mathbf{6 a}-\mathbf{i}$.

$N^{3}, N^{11}$-Bis(2-hydroxyethyl)-14-phenyl-14H-dibenzo[a,j]xanthene-3,11-dicarboxamide (6a): White solid. Yield $88.9 \%$. $\mathrm{mp} \quad 202-204^{\circ} \mathrm{C}$. ${ }^{1} \mathrm{H}-\mathrm{NMR}$ (DMSO- $\left.d_{6}, \quad 300 \mathrm{MHz}\right) \quad \delta: 8.75$ (d, $J=9.0 \mathrm{~Hz}, 2 \mathrm{H}, 1,13-\mathrm{H}), 8.61(\mathrm{t}, J=5.2 \mathrm{~Hz}, 2 \mathrm{H}, \mathrm{NH} \times 2)$, $8.48(\mathrm{~s}, 2 \mathrm{H}, 4,10-\mathrm{H}), 8.05(\mathrm{~m}, 4 \mathrm{H}, 5,9,2,12-\mathrm{H}), 7.64(\mathrm{~m}$, $\left.4 \mathrm{H}, 6,8,2^{\prime}, 6^{\prime}-\mathrm{H}\right), 7.15\left(\mathrm{t}, J=7.5 \mathrm{~Hz}, 2 \mathrm{H}, 3^{\prime}, 5^{\prime}-\mathrm{H}\right), 6.98(\mathrm{t}$, $\left.J=7.2 \mathrm{~Hz}, 1 \mathrm{H}, 4^{\prime}-\mathrm{H}\right), 6.79$ (s, 1H, 14-H), 4.79 (s, 2H, OH $\left.\times 2\right)$, 3.62-3.45 (m, 4H, $\left.\mathrm{NHCH}_{2} \mathrm{CH}_{2} \mathrm{OH} \times 2\right), 3.44-3.31(\mathrm{~m}, 4 \mathrm{H}$, $\mathrm{NHCH}_{2} \mathrm{CH}_{2} \mathrm{OH} \times 2$ ). ${ }^{13} \mathrm{C}-\mathrm{NMR}$ (DMSO- $\left.d_{6}, 75 \mathrm{MHz}\right) \delta: 166.6$, $149.4,145.8,132.7,131.1,130.6,130.4,128.9,128.7,128.4$, $126.9,125.6,124.1,118.9,117.9,60.3,42.8,36.9$. IR (KBr) v: 2929, 2876, 1638, 1546, 1462, 1399, $1249 \mathrm{~cm}^{-1}$. HR-MS (ESI) Calcd for $\mathrm{C}_{33} \mathrm{H}_{29} \mathrm{~N}_{2} \mathrm{O}_{5}[\mathrm{M}+\mathrm{H}]^{+}$533.2076, Found 533.2070.

$N^{3}, N^{11}$-Bis(2-hydroxyethyl)-14-(2-fluorophenyl)-14H-dibenzo $[a, j]$ xanthene-3,11-dicarboxamide (6b): White solid. Yield 90.7\%. mp 201-203 ${ }^{\circ} \mathrm{C} .{ }^{1} \mathrm{H}-\mathrm{NMR}$ (DMSO- $d_{6}, 300 \mathrm{MHz}$ ) $\delta$ : $8.58(\mathrm{t}, J=5.6 \mathrm{~Hz}, 2 \mathrm{H}, \mathrm{NH} \times 2), 8.49(\mathrm{~s}, 2 \mathrm{H}, 4,10-\mathrm{H}), 8.47$ (d, $J=8.9 \mathrm{~Hz}, 2 \mathrm{H}, 1,13-\mathrm{H}), 8.07$ (d, $J=9.0 \mathrm{~Hz}, 2 \mathrm{H}, 5,9-\mathrm{H})$, $8.05(\mathrm{dd}, J=8.9,1.5 \mathrm{~Hz}, 2 \mathrm{H}, 2,12-\mathrm{H}), 7.63(\mathrm{~d}, J=9.0 \mathrm{~Hz}$, $2 \mathrm{H}, 6,8-\mathrm{H}), 7.64-7.54(\mathrm{~m}, 1 \mathrm{H}, \mathrm{Ar}-\mathrm{H}), 7.13-6.97(\mathrm{~m}, 3 \mathrm{H}$, Ar-H), $6.90(\mathrm{~s}, 1 \mathrm{H}, 14-\mathrm{H}), 4.76(\mathrm{t}, J=5.6 \mathrm{~Hz}, 2 \mathrm{H}, \mathrm{OH} \times 2)$, 3.54 (q, $\left.J=6.0 \mathrm{~Hz}, 4 \mathrm{H}, \mathrm{NHCH}_{2} \mathrm{CH}_{2} \mathrm{OH} \times 2\right), 3.40-3.34(\mathrm{~m}, 4 \mathrm{H}$, $\left.\mathrm{NHCH}_{2} \mathrm{CH}_{2} \mathrm{OH} \times 2\right) .{ }^{13} \mathrm{C}-\mathrm{NMR}\left(\mathrm{DMSO}-d_{6}, 75 \mathrm{MHz}\right) \delta: 166.5$, $159.0\left({ }^{1} J_{\mathrm{CF}}=242.7 \mathrm{~Hz}\right), 149.6,132.6,131.9\left({ }^{2} J_{\mathrm{CF}}=13.1 \mathrm{~Hz}\right), 131.4$, 131.1, 131.0, 130.3, $129.5\left({ }^{3} J_{\mathrm{CF}}=8.4 \mathrm{~Hz}\right), 128.9,125.8,125.7$ $\left({ }^{2} J_{\mathrm{CF}}=21.5 \mathrm{~Hz}\right), 123.0,118.9,116.2\left({ }^{2} J_{\mathrm{CF}}=22.4 \mathrm{~Hz}\right), 115.7,60.3$, 42.8, 31.6. IR (KBr) v: 2938, 2880, 1640, 1547, 1463, 1401, $1252 \mathrm{~cm}^{-1}$. HR-MS (ESI) Calcd for $\mathrm{C}_{33} \mathrm{H}_{28} \mathrm{FN}_{2} \mathrm{O}_{5}[\mathrm{M}+\mathrm{H}]^{+}$ 551.1982, Found 551.1989. 
$N^{3}, N^{11}$-Bis(2-hydroxyethyl)-14-(4-fluorophenyl)-14Hdibenzo[a,j]xanthene-3,11-dicarboxamide (6c): White solid. Yield $92.0 \%$. mp $268-269^{\circ} \mathrm{C} .{ }^{1} \mathrm{H}-\mathrm{NMR}$ (DMSO- $d_{6}, 300 \mathrm{MHz}$ ) $\delta: 8.75(\mathrm{~d}, J=9.0 \mathrm{~Hz}, 2 \mathrm{H}, 1,13-\mathrm{H}), 8.62(\mathrm{t}, J=5.5 \mathrm{~Hz}, 2 \mathrm{H}$, $\mathrm{NH} \times 2), 8.49($ brs, 2H, 4, 10-H), 8.07-8.03 (m, 4H, 5, 9, 2, 12-H), 7.69-7.61 (m, 4H, 6, 8, 2', 6'-H), 6.98 (t, $J=8.8 \mathrm{~Hz}, 2 \mathrm{H}$, $\left.3^{\prime}, 5^{\prime}-\mathrm{H}\right), 6.83(\mathrm{~s}, 1 \mathrm{H}, 14-\mathrm{H}), 4.79(\mathrm{t}, J=5.1 \mathrm{~Hz}, 2 \mathrm{H}, \mathrm{OH} \times 2)$, 3.64-3.50 (m, 4H, $\left.\mathrm{NHCH}_{2} \mathrm{CH}_{2} \mathrm{OH} \times 2\right), 3.45-3.34(\mathrm{~m}, 4 \mathrm{H}$, $\left.\mathrm{NHCH}_{2} \mathrm{CH}_{2} \mathrm{OH} \times 2\right) .{ }^{13} \mathrm{C}-\mathrm{NMR}\left(\mathrm{DMSO}-d_{6}, 75 \mathrm{MHz}\right) \delta: 166.6$, $161.0\left({ }^{1} J_{\mathrm{CF}}=242.3 \mathrm{~Hz}\right), 149.4,142.0,132.6,131.1,130.8,130.4$, $130.2\left({ }^{3} J_{\mathrm{CF}}=8.1 \mathrm{~Hz}\right), 128.8,125.6,124.0,119.0,117.8,115.7$ $\left({ }^{2} J_{\mathrm{CF}}=21.0 \mathrm{~Hz}\right), 60.3,42.8,36.0$. IR (KBr) $v: 2933,2876,1624$, $1549,1506,1463,1400,1250 \mathrm{~cm}^{-1}$. HR-MS (ESI) Calcd for $\mathrm{C}_{33} \mathrm{H}_{28} \mathrm{FN}_{2} \mathrm{O}_{5}[\mathrm{M}+\mathrm{H}]^{+}$551.1982, Found 551.1974.

$N^{3}, N^{11}$-Bis(2-hydroxyethyl)-14-(2-chlorophenyl)-14Hdibenzo[a,j]xanthene-3,11-dicarboxamide (6d): White solid. Yield $85.5 \%$. mp $295-297^{\circ} \mathrm{C} .{ }^{1} \mathrm{H}-\mathrm{NMR}$ (DMSO- $d_{6}, 300 \mathrm{MHz}$ ) $\delta: 8.64-8.56(\mathrm{~m}, 4 \mathrm{H}, 1,13-\mathrm{H}, \mathrm{NH} \times 2), 8.47(\mathrm{~d}, J=1.3 \mathrm{~Hz}, 2 \mathrm{H}$, 4, 10-H), 8.07-8.04 (m, 4H, 5, 9, 2, 12-H), 7.61 (d, $J=8.9 \mathrm{~Hz}$, $2 \mathrm{H}, 6,8-\mathrm{H}), 7.51$ (brd, $\left.J=7.8 \mathrm{~Hz}, 1 \mathrm{H}, 6^{\prime}-\mathrm{H}\right), 7.32$ (dd, $J=7.9$, $\left.1.2 \mathrm{~Hz}, 1 \mathrm{H}, 3^{\prime}-\mathrm{H}\right), 7.16-7.00\left(\mathrm{~m}, 2 \mathrm{H}, 4^{\prime}, 5^{\prime}-\mathrm{H}\right), 6.84(\mathrm{~s}, 1 \mathrm{H}$, $14-\mathrm{H}), 4.75$ (t, $J=5.6 \mathrm{~Hz}, 2 \mathrm{H}, \mathrm{OH} \times 2), 3.54$ (q, $J=6.0 \mathrm{~Hz}, 4 \mathrm{H}$, $\left.\mathrm{NHCH}_{2} \mathrm{CH}_{2} \mathrm{OH} \times 2\right), \quad 3.38-3.34\left(\mathrm{~m}, 4 \mathrm{H}, \quad \mathrm{NHCH}_{2} \mathrm{CH}_{2} \mathrm{OH} \times 2\right)$. ${ }^{13} \mathrm{C}-\mathrm{NMR}$ (DMSO- $\left.d_{6}, 75 \mathrm{MHz}\right) \delta: 166.5,149.7,142.8,132.8$, $132.3,131.1,131.1,130.6,130.5,130.3,129.2,128.9,128.7$, $125.6,123.6,119.0,116.7,60.3,42.8,35.2$. IR (KBr) v: 2929, 2868, 1645, 1548, 1464, 1399, $1254 \mathrm{~cm}^{-1}$. HR-MS (ESI) Calcd for $\mathrm{C}_{33} \mathrm{H}_{28} \mathrm{ClN}_{2} \mathrm{O}_{5}[\mathrm{M}+\mathrm{H}]^{+}$567.1687, Found 567.1690.

$N^{3}, N^{11}$-Bis(2-hydroxyethyl)-14-(4-chlorophenyl)-14Hdibenzo[a,j]xanthene-3,11-dicarboxamide (6e): White solid. Yield $91.5 \%$. mp $202-203^{\circ} \mathrm{C}$. ${ }^{1} \mathrm{H}-\mathrm{NMR}$ (DMSO- $d_{6}, 300 \mathrm{MHz}$ ) $\delta: 8.72(\mathrm{~d}, J=9.0 \mathrm{~Hz}, 2 \mathrm{H}, 1,13-\mathrm{H}), 8.60(\mathrm{t}, J=5.5 \mathrm{~Hz}, 2 \mathrm{H}$, $\mathrm{NH} \times 2), 8.47(\mathrm{~s}, 2 \mathrm{H}, 4,10-\mathrm{H}), 8.06-8.03(\mathrm{~m}, 4 \mathrm{H}, 5,9,2$, 12-H), 7.65-7.61 (m, 4H, 6, 8, 2', 6'-H), 7.21(d, $J=8.5 \mathrm{~Hz}, 2 \mathrm{H}$, $\left.3^{\prime}, 5^{\prime}-\mathrm{H}\right), 6.81(\mathrm{~s}, 1 \mathrm{H}, 14-\mathrm{H}), 4.76(\mathrm{t}, J=5.5 \mathrm{~Hz}, 2 \mathrm{H}, \mathrm{OH} \times 2)$, 3.56-3.50 (m, 4H, $\left.\mathrm{NHCH}_{2} \mathrm{CH}_{2} \mathrm{OH} \times 2\right), 3.40-3.34(\mathrm{~m}, 4 \mathrm{H}$, $\mathrm{NHCH}_{2} \mathrm{CH}_{2} \mathrm{OH} \times 2$ ). ${ }^{13} \mathrm{C}-\mathrm{NMR}$ (DMSO- $\left.d_{6}, 75 \mathrm{MHz}\right) \delta: 166.6$, $149.4,144.7,132.6,131.5,131.1,130.9,130.4,130.1,128.9$, $128.8,125.7,123.9,118.9,117.4,60.3,42.8,36.2$. IR (KBr) $v$ : 2921, 2864, 1638, 1624, 1547, 1462, 1399, $1251 \mathrm{~cm}^{-1}$. HR-MS (ESI) Calcd for $\mathrm{C}_{33} \mathrm{H}_{28} \mathrm{ClN}_{2} \mathrm{O}_{5}[\mathrm{M}+\mathrm{H}]^{+}$567.1687, Found 567.1693 .

$N^{3}, N^{11}$-Bis(2-hydroxyethyl)-14-(4-bromophenyl)-14Hdibenzo[a,j]xanthene-3,11-dicarboxamide (6f): White solid. Yield $88.8 \%$. mp $252-254^{\circ} \mathrm{C} .{ }^{1} \mathrm{H}-\mathrm{NMR}$ (DMSO- $d_{6}, 300 \mathrm{MHz}$ ) $\delta: 8.73(\mathrm{~d}, J=9.0 \mathrm{~Hz}, 2 \mathrm{H}, 1,13-\mathrm{H}), 8.62(\mathrm{t}, J=5.5 \mathrm{~Hz}, 2 \mathrm{H}$, $\mathrm{NH} \times 2), 8.49(\mathrm{~s}, 2 \mathrm{H}, 4,10-\mathrm{H}), 8.07-8.04(\mathrm{~m}, 4 \mathrm{H}, 5,9,2$, $12-\mathrm{H}), 7.63(\mathrm{~d}, J=9.0 \mathrm{~Hz}, 2 \mathrm{H}, 6,8-\mathrm{H}), 7.59(\mathrm{~d}, J=8.4 \mathrm{~Hz}$, $\left.2 \mathrm{H}, 2^{\prime}, 6^{\prime}-\mathrm{H}\right), 7.35$ (d, J=8.2 Hz, 2H, 3', 5'-H), 6.81 (s, 1H, $14-\mathrm{H}), 4.78(\mathrm{t}, J=5.5 \mathrm{~Hz}, 2 \mathrm{H}, \mathrm{OH} \times 2), 3.58-3.52(\mathrm{~m}, 4 \mathrm{H}$, $\left.\mathrm{NHCH}_{2} \mathrm{CH}_{2} \mathrm{OH} \times 2\right), \quad 3.42-3.36\left(\mathrm{~m}, 4 \mathrm{H}, \quad \mathrm{NHCH}_{2} \mathrm{CH}_{2} \mathrm{OH} \times 2\right)$. ${ }^{13} \mathrm{C}-\mathrm{NMR}$ (DMSO- $\left.d_{6}, 75 \mathrm{MHz}\right) \delta$ : 166.6, 149.3, 145.1, 132.6, $131.9,131.1,130.9,130.5,130.4,128.8,125.7,123.9,120.1$, $118.9,117.4,60.3,42.8,36.3$. IR (KBr) v: 2929, 2884, 1642, 1622, 1539, 1464, 1400, 1256 $\mathrm{cm}^{-1}$. HR-MS (ESI) Calcd for $\mathrm{C}_{33} \mathrm{H}_{28} \mathrm{BrN}_{2} \mathrm{O}_{5}[\mathrm{M}+\mathrm{H}]^{+}$611.1182, Found 611.1184.

$N^{3}, N^{11}$-Bis(2-hydroxyethyl)-14-(3-nitrophenyl)-14Hdibenzo[a,j]xanthene-3,11-dicarboxamide (6g): White solid. Yield 88.9\%. mp $299-300^{\circ} \mathrm{C} .{ }^{1} \mathrm{H}-\mathrm{NMR}$ (DMSO- $d_{6}, 300 \mathrm{MHz}$ ) $\delta: 8.82(\mathrm{~d}, J=9.0 \mathrm{~Hz}, 2 \mathrm{H}, 1,13-\mathrm{H}), 8.71-8.54\left(\mathrm{~m}, 3 \mathrm{H}, 2^{\prime}-\mathrm{H}\right.$, $\mathrm{NH} \times 2), 8.49(\mathrm{~d}, J=1.5 \mathrm{~Hz}, 2 \mathrm{H}, 4,10-\mathrm{H}), 8.10-8.05(\mathrm{~m}, 5 \mathrm{H}$,
5, 9, 2, 12, 6'-H), 7.87 (dd, $\left.J=8.2,1.6 \mathrm{~Hz}, 1 \mathrm{H}, 4^{\prime}-\mathrm{H}\right), 7.68$ (d, $J=8.9 \mathrm{~Hz}, 2 \mathrm{H}, 6,8-\mathrm{H}), 7.47$ (t, $\left.J=8.0 \mathrm{~Hz}, 1 \mathrm{H}, 5^{\prime}-\mathrm{H}\right)$, $7.04(\mathrm{~s}, 1 \mathrm{H}, 14-\mathrm{H}), 4.77(\mathrm{t}, 2 \mathrm{H}, J=5.6 \mathrm{~Hz}, \mathrm{OH} \times 2), 3.54$ (q, $\left.4 \mathrm{H}, J=6.0 \mathrm{~Hz}, \quad \mathrm{NHCH}_{2} \mathrm{CH}_{2} \mathrm{OH} \times 2\right), 3.46-3.21 \quad(\mathrm{~m}, 4 \mathrm{H}$, $\left.\mathrm{NHCH}_{2} \mathrm{CH}_{2} \mathrm{OH} \times 2\right) .{ }^{13} \mathrm{C}-\mathrm{NMR}\left(\mathrm{DMSO}-d_{6}, 75 \mathrm{MHz}\right) \delta: 166.5$, $149.5,148.3,147.7,134.8,132.5,131.3,131.3,130.7,130.5$, $128.9,125.8,123.8,122.5,122.2,119.0,116.9,60.2,42.8$, 36.3. IR (KBr) v: 2938, 2884, 1647, 1622, 1536, 1462, 1398, $1357,1253 \mathrm{~cm}^{-1}$. HR-MS (ESI) Calcd for $\mathrm{C}_{33} \mathrm{H}_{28} \mathrm{~N}_{3} \mathrm{O}_{7}[\mathrm{M}+\mathrm{H}]^{+}$ 578.1927, Found 578.1929.

$N^{3}, N^{11}$-Bis(2-hydroxyethyl)-14-(4-nitrophenyl)-14Hdibenzo $[a, j]$ xanthene-3,11-dicarboxamide (6h): White solid. Yield $90.5 \%$. mp $228-230^{\circ} \mathrm{C} .{ }^{1} \mathrm{H}-\mathrm{NMR}$ (DMSO- $d_{6}, 300 \mathrm{MHz}$ ) $\delta: 8.75(\mathrm{~d}, J=8.4 \mathrm{~Hz}, 2 \mathrm{H}, 1,13-\mathrm{H}), 8.63$ (brs, $2 \mathrm{H}, \mathrm{NH} \times 2)$, 8.49 (s, 2H, 4, 10-H), 8.15-7.98 (m, 6H, 5, 9, 2, 12, 2', 6'-H), $7.94\left(\mathrm{~d}, J=7.2 \mathrm{~Hz}, 2 \mathrm{H}, 3^{\prime}, 5^{\prime}-\mathrm{H}\right), 7.66(\mathrm{~d}, J=8.7 \mathrm{~Hz}, 2 \mathrm{H}, 6$, $8-\mathrm{H}), 7.00$ (s, 1H, 14-H), 4.79 (brs, 2H, OH $\times 2), 3.54$ (s, $\left.4 \mathrm{H}, \quad \mathrm{NHCH}_{2} \mathrm{CH}_{2} \mathrm{OH} \times 2\right), 3.38\left(\mathrm{~s}, 4 \mathrm{H}, \quad \mathrm{NHCH}_{2} \mathrm{CH}_{2} \mathrm{OH} \times 2\right)$. ${ }^{13} \mathrm{C}-\mathrm{NMR}\left(\mathrm{DMSO}-d_{6}, 75 \mathrm{MHz}\right) \delta: 166.5,152.8,149.4,146.4$, $132.5,131.3,131.3,130.4,129.5,128.8,125.8,124.3,123.8$, 119.0, 116.7, 60.3, 42.8, 36.7. IR (KBr) v: 2938, 2880, 1640, $1546,1516,1462,1343,1253 \mathrm{~cm}^{-1}$. HR-MS (ESI) Calcd for $\mathrm{C}_{33} \mathrm{H}_{28} \mathrm{~N}_{3} \mathrm{O}_{7}[\mathrm{M}+\mathrm{H}]^{+}$578.1927, Found 578.1933.

$N^{3}, N^{11}$-Bis(2-hydroxyethyl)-14-(4-methylphenyl)-14Hdibenzo $[a, j]$ xanthene-3,11-dicarboxamide (6i): White solid. Yield $89.8 \%$. mp $>300{ }^{\circ} \mathrm{C}$. ${ }^{1} \mathrm{H}-\mathrm{NMR}\left(\mathrm{DMSO}-d_{6}, 300 \mathrm{MHz}\right)$ $\delta: 8.72(\mathrm{~d}, J=8.8 \mathrm{~Hz}, 2 \mathrm{H}, 1,13-\mathrm{H}), 8.60$ (brs, $2 \mathrm{H}, \mathrm{NH} \times 2)$, 8.47 (s, 2H, 4, 10-H), 8.12-7.99 (m, 4H, 5, 9, 2, 12-H), 7.62 (d, $J=8.8 \mathrm{~Hz}, 2 \mathrm{H}, 6,8-\mathrm{H}), 7.49$ (d, J=7.6 Hz, 2H, 2', 6'-H), $6.93\left(\mathrm{~d}, J=7.5 \mathrm{~Hz}, 2 \mathrm{H}, 3^{\prime}, 5^{\prime}-\mathrm{H}\right), 6.74(\mathrm{~s}, 1 \mathrm{H}, 14-\mathrm{H}), 4.78(\mathrm{t}$, $J=5.1 \mathrm{~Hz}, 2 \mathrm{H}, \mathrm{OH} \times 2), 3.62-3.50\left(\mathrm{~m}, 4 \mathrm{H}, \mathrm{NHCH}_{2} \mathrm{CH}_{2} \mathrm{OH} \times 2\right)$, 3.43-3.32 (m, 4H, $\left.\mathrm{NHCH}_{2} \mathrm{CH}_{2} \mathrm{OH} \times 2\right), 2.04$ (s, 3H, $\left.\mathrm{CH}_{3}\right)$. ${ }^{13} \mathrm{C}-\mathrm{NMR}$ (DMSO- $\left.d_{6}, 7 \overline{5 \mathrm{MHz}}\right) \delta$ : 166.6, 149.3, 142.9, 136.0, $132.7,131.0,130.5,130.4,129.4,128.7,128.3,125.5,124.1$, $118.9,118.0,60.3,42.8,36.5,20.8$. IR (KBr) v: 2921, 2848, 1644, 1556, 1536, 1401, $1252 \mathrm{~cm}^{-1}$. HR-MS (ESI) Calcd for $\mathrm{C}_{34} \mathrm{H}_{31} \mathrm{~N}_{2} \mathrm{O}_{5}[\mathrm{M}+\mathrm{H}]^{+}$547.2233, Found 547.2228.

General Procedure for the Preparation of $N^{3}, N^{11}$-Bis(2hydroxyethyl)-14-aryl-14H-dibenzo $[a, j]$ xanthene-3,11dimethanol $(\mathbf{8 a}, \mathbf{8 e}, \mathbf{8 f})$ The acid chloride of $4 \mathrm{mmol}$ dicarboxylic acid $\mathbf{5}$ was prepared with the former method, and it was added in portions to a solution of $0.3 \mathrm{~g}(8 \mathrm{mmol})$ of lithium aluminum hydride in $20 \mathrm{~mL}$ of anhydrous THF. The mixture was stirred at $0^{\circ} \mathrm{C}$ for $15 \mathrm{~min}$. Decomposition was carried out with water and then with $10 \mathrm{~mL}$ of $10 \% \mathrm{NaOH}$ to dissovle the precipitation and the mixed solution was extracted three times with EtOAc $(3 \times 20 \mathrm{~mL})$ and the collected EtOAc layers were washed with brine and water successively and then dried $\left(\mathrm{MgSO}_{4}\right)$, filtered and concentrated to give compounds $8 \mathbf{a}, \mathbf{8 e}$, and $\mathbf{8 f}$.

$N^{3}, N^{11}$-Bis(2-hydroxyethyl)-14-phenyl-14H-dibenzo[a,j]xanthene-3,11-dimethanol (8a): White solid. Yield 91.2\%. mp $216-218^{\circ} \mathrm{C}$. ${ }^{1} \mathrm{H}-\mathrm{NMR}$ (DMSO- $\left.d_{6}, 300 \mathrm{MHz}\right) \delta$ : $8.62(\mathrm{~d}$, $J=8.8 \mathrm{~Hz}, 2 \mathrm{H}, 1,13-\mathrm{H}), 7.89$ (d, $J=8.9 \mathrm{~Hz}, 2 \mathrm{H}, 5,9-\mathrm{H}), 7.83$ (brs, 2H, 4, 10-H), 7.60-7.55 (m, 4H, 2, 12, 2', 6'-H), 7.52 (d, $J=8.9 \mathrm{~Hz}, 2 \mathrm{H}, 6,8-\mathrm{H}), 7.12\left(\mathrm{t}, J=7.7 \mathrm{~Hz}, 2 \mathrm{H}, 3^{\prime}, 5^{\prime}-\mathrm{H}\right), 6.95$ $\left(\mathrm{t}, J=7.7 \mathrm{~Hz}, 1 \mathrm{H}, 4^{\prime}-\mathrm{H}\right), 6.68(\mathrm{~s}, 1 \mathrm{H}, 14-\mathrm{H}), 5.26(\mathrm{t}, J=5.7 \mathrm{~Hz}$, $\left.2 \mathrm{H}, \mathrm{CH}_{2} \mathrm{OH} \times 2\right), 4.64\left(\mathrm{~d}, J=5.6 \mathrm{~Hz}, 4 \mathrm{H}, \mathrm{CH}_{2} \mathrm{OH} \times 2\right) .{ }^{13} \mathrm{C}-\mathrm{NMR}$ (DMSO- $\left.d_{6}, 75 \mathrm{MHz}\right) \delta: 148.2,146.1,139.2,131.0,130.4,129.3$, $128.8,128.4,126.7,126.7,125.8,123.8,118.1,117.9,63.3,37.1$. IR $(\mathrm{KBr}) v: 1600,1467,1401,1253,1243,1039 \mathrm{~cm}^{-1}$. HR-MS 
(ESI) Calcd for $\mathrm{C}_{29} \mathrm{H}_{23} \mathrm{O}_{3}[\mathrm{M}+\mathrm{H}]^{+}$419.1647, Found 419.1658.

$N^{3}, N^{11}$-Bis(2-hydroxyethyl)-14-(4-chlorophenyl)-14Hdibenzo $[a, j]$ xanthene-3,11-dimethanol $(\mathbf{8 e})$ : White solid. Yield 92.1\%. mp $222-224^{\circ} \mathrm{C} .{ }^{1} \mathrm{H}-\mathrm{NMR}$ (DMSO- $\left.d_{6}, 300 \mathrm{MHz}\right) \delta: 8.59$ (d, $J=8.7 \mathrm{~Hz}, 2 \mathrm{H}, 1,13-\mathrm{H}), 7.89$ (d, $J=9.0 \mathrm{~Hz}, 2 \mathrm{H}, 5,9-\mathrm{H}), 7.83$ (brs, 2H, 4, 10-H), 7.60 (d, $\left.J=8.5 \mathrm{~Hz}, 2 \mathrm{H}, 2^{\prime}, 66^{\prime}-\mathrm{H}\right), 7.56$ (brd, $J=8.7 \mathrm{~Hz}, 2 \mathrm{H}, 2,12-\mathrm{H}), 7.51$ (d, $J=9.0 \mathrm{~Hz}, 2 \mathrm{H}, 6,8-\mathrm{H}), 7.18$ (d, $\left.J=8.4 \mathrm{~Hz}, 3^{\prime}, 5^{\prime}-\mathrm{H}\right), 6.70(\mathrm{~s}, 1 \mathrm{H}, 14-\mathrm{H}), 5.25(\mathrm{t}, J=5.7 \mathrm{~Hz}$, $\left.2 \mathrm{H}, \mathrm{CH}_{2} \mathrm{OH} \times 2\right), 4.63$ (d, $\left.J=5.6 \mathrm{~Hz}, 4 \mathrm{H}, \mathrm{CH}_{2} \mathrm{OH} \times 2\right) .{ }^{13} \mathrm{C}-\mathrm{NMR}$ (DMSO-d, $75 \mathrm{MHz}) \delta: 148.2,145.0,139.3,131.3,131.0,130.3$, $130.1,129.5,128.8,126.8,125.9,123.6,118.1,117.4,63.2$, 36.4. IR (KBr) v: 1598, 1489, 1466, 1400, 1252, $1015 \mathrm{~cm}^{-1}$. HR-MS (ESI) Calcd for $\mathrm{C}_{29} \mathrm{H}_{22} \mathrm{ClO}_{3}[\mathrm{M}+\mathrm{H}]^{+}$453.1257, Found 453.1250 .

$N^{3}, N^{11}$-Bis(2-hydroxyethyl)-14-(4-bromophenyl)-14Hdibenzo $[a, j]$ xanthene-3,11-dimethanol (8f): White solid. Yield 89.1\%. mp $230-232^{\circ} \mathrm{C} .{ }^{1} \mathrm{H}-\mathrm{NMR}\left(\mathrm{CDCl}_{3}, 300 \mathrm{MHz}\right) \delta: 8.28$ (d, $J=8.7 \mathrm{~Hz}, 2 \mathrm{H}, 1,13-\mathrm{H}), 7.78$ (s, 2H, 4, 10-H), 7.77 (d, $J=8.8 \mathrm{~Hz}, 2 \mathrm{H}, 5,9-\mathrm{H}), 7.57$ (dd, $J=8.7,1.7 \mathrm{~Hz}, 2 \mathrm{H}, 2,12-\mathrm{H})$, $7.47(\mathrm{~d}, J=8.9 \mathrm{~Hz}, 2 \mathrm{H}, 6,8-\mathrm{H}), 7.36$ (d, $J=8.5 \mathrm{~Hz}, 2 \mathrm{H}, 2^{\prime}$, $\left.6^{\prime}-\mathrm{H}\right), 7.23$ (d, J=8.5 Hz, 3', 5'-H), 6.40 (s, 1H, 14-H), 4.82 (s, $\left.4 \mathrm{H}, \underline{\mathrm{CH}}_{2} \mathrm{OH} \times 2\right) 1.96\left(\mathrm{~s}, 2 \mathrm{H}, \mathrm{CH}_{2} \mathrm{OH} \times 2\right) .{ }^{13} \mathrm{C}-\mathrm{NMR}\left(\mathrm{CDCl}_{3}\right.$, $75 \mathrm{MHz}) \delta$ : 148.6, 143.9, 136.9, 131.6, 131.0, 130.7, 129.8, 129.1, $126.6,126.3,122.9,120.3,118.3,116.6,65.2,37.6$. IR (KBr) $v$ : $1597,1485,1466,1400,1253,1242,1010 \mathrm{~cm}^{-1}$. HR-MS (ESI) Calcd for $\mathrm{C}_{29} \mathrm{H}_{22} \mathrm{BrO}_{3}[\mathrm{M}+\mathrm{H}]^{+}$497.0752, Found 497.0760.

General Procedure for the Preparation of $N^{3}, N^{11}$-Bis(2hydroxyethyl)-14-aryl-14H-dibenzo $[a, j]$ xanthene-3,11dimethanamine (9a, 9e, 9f) A $100 \mathrm{~mL}$, three-necked flask, equipped with a magnetic stir bar, a condenser, a thermometer and an addition funnel, was charged with $2 \mathrm{mmol}$ compounds $\mathbf{8}, 20 \mathrm{~mL}$ of $\mathrm{CHCl}_{3}$ and 1 drop of dimethylformamide. Afterwards a solution of $1 \mathrm{~mL}(13.6 \mathrm{mmol})$ thionyl chloride in $\mathrm{CHCl}_{3}(4 \mathrm{~mL})$ was added, and the mixture was stirred at room temperature for $0.5 \mathrm{~h}$. After the solvent was removed by evacuation under reduced pressure, a yellow solid can be obtained. $\mathrm{A} \mathrm{CHCl}_{3}(10 \mathrm{~mL})$ solution of the yellow solid was then added to the solution of 2-aminoethanol (488 $\mathrm{mg}, 8 \mathrm{mmol}$ ) in $\mathrm{CHCl}_{3}(25 \mathrm{~mL})$. The mixture was then stirred for $6 \mathrm{~h}$ at room temperature. The $\mathrm{CHCl}_{3}$ solution was washed with brine and water successively and then dried $\left(\mathrm{MgSO}_{4}\right)$, filtered and concentrated. The crude products were recrystallized from petroleum ether-EtOAc $(3: 1)$ to give compounds $9 \mathbf{a}, \mathbf{9 e}$, and $\mathbf{9 f}$.

$N^{3}, N^{11}$-Bis(2-hydroxyethyl)-14-phenyl-14H-dibenzo[a,j]xanthene-3,11-dimethanamine (9a): White solid. Yield 81.2\%. mp $118-120^{\circ} \mathrm{C} .{ }^{1} \mathrm{H}-\mathrm{NMR}\left(\mathrm{CDCl}_{3}, 300 \mathrm{MHz}\right) \delta: 8.31$ (d, $J=$ $9.0 \mathrm{~Hz}, 2 \mathrm{H}, 1,13-\mathrm{H}), 7.72(\mathrm{~d}, J=8.7 \mathrm{~Hz}, 2 \mathrm{H}, 5,9-\mathrm{H}), 7.68$ (brs, 2H, 4, 10-H), 7.54-7.44 (m, 6H, 6, 8, 2, 12, 2', 6'-H), 7.11 (t, $\left.J=7.5 \mathrm{~Hz}, 2 \mathrm{H}, 3^{\prime}, 5^{\prime}-\mathrm{H}\right), 6.95$ (t, $\left.J=7.5 \mathrm{~Hz}, 1 \mathrm{H}, 4^{\prime}-\mathrm{H}\right)$, $6.38(\mathrm{~s}, 1 \mathrm{H}, 14-\mathrm{H}), 3.90\left(\mathrm{~s}, 4 \mathrm{H}, \mathrm{CH}_{2} \mathrm{NHCH}_{2} \mathrm{CH}_{2} \mathrm{OH} \times 2\right)$, $3.66\left(\mathrm{t}, \quad J=5.1 \mathrm{~Hz}, \quad 4 \mathrm{H}, \quad \mathrm{CH}_{2} \mathrm{NHCH}_{2} \mathrm{CH}_{2} \mathrm{OH} \times 2\right), 2.80 \quad(\mathrm{t}$, $J=5.1 \mathrm{~Hz}, \quad 4 \mathrm{H}, \quad \mathrm{CH}_{2} \mathrm{NHCH}_{2} \mathrm{CH}_{2} \mathrm{OH} \times 2$ ), 2.62 (brs, 4H, $\left.\mathrm{CH}_{2} \mathrm{NHCH}_{2} \mathrm{CH}_{2} \mathrm{OH} \times 2\right) .{ }^{13} \mathrm{C}-\mathrm{NMR}\left(\mathrm{CDCl}_{3}, 75 \mathrm{MHz}\right) \delta: 148.6$, $145.0,135.3,131.0,130.7,128.6,128.5,128.2,127.7,127.4$, $126.4,123.1,118.2,117.2,60.8,53.2,50.5,38.2$. IR (KBr) v: 2923, 2852, 1597, 1466, 1400, 1252, $1242 \mathrm{~cm}^{-1}$. HR-MS (ESI) Calcd for $\mathrm{C}_{33} \mathrm{H}_{33} \mathrm{~N}_{2} \mathrm{O}_{3}[\mathrm{M}+\mathrm{H}]^{+}$505.2491, Found 505.2483.

$N^{3}, N^{11}$-Bis(2-hydroxyethyl)-14-(4-chlorophenyl)-14Hdibenzo[a,j]xanthene-3,11-dimethanamine (9e): White solid. Yield 79.2\%. mp $119-121^{\circ} \mathrm{C} .{ }^{1} \mathrm{H}-\mathrm{NMR}\left(\mathrm{CDCl}_{3}, 300 \mathrm{MHz}\right)$ $\delta: 8.25(\mathrm{~d}, J=8.7 \mathrm{~Hz}, 2 \mathrm{H}, 1,13-\mathrm{H}), 7.75(\mathrm{~d}, J=9.0 \mathrm{~Hz}, 2 \mathrm{H}$,
5, 9-H), 7.72 (brs, 2H, 4, 10-H), 7.54 (dd, $J=8.7,1.6 \mathrm{~Hz}$, $2 \mathrm{H}, 2,12-\mathrm{H}), 7.46(\mathrm{~d}, J=9.0 \mathrm{~Hz}, 2 \mathrm{H}, 6,8-\mathrm{H}), 7.42(\mathrm{~d}$, $\left.J=8.4 \mathrm{~Hz}, 2 \mathrm{H}, 2^{\prime}, 6^{\prime}-\mathrm{H}\right), 7.08\left(\mathrm{~d}, J=8.4 \mathrm{~Hz}, 2 \mathrm{H}, 3^{\prime}, 5^{\prime}-\mathrm{H}\right)$, $6.39(\mathrm{~s}, 1 \mathrm{H}, 14-\mathrm{H}), 3.93\left(\mathrm{~s}, 4 \mathrm{H}, \mathrm{CH}_{2} \mathrm{NHCH}_{2} \mathrm{CH}_{2} \mathrm{OH} \times 2\right)$, $3.68\left(\mathrm{t}, \quad J=5.1 \mathrm{~Hz}, \quad 4 \mathrm{H}, \quad \mathrm{CH}_{2} \mathrm{NHCH}_{2} \mathrm{CH}_{2} \mathrm{OH} \times 2\right), 2.83$ (t, $\left.J=5.1 \mathrm{~Hz}, \quad 4 \mathrm{H}, \quad \mathrm{CH}_{2} \mathrm{NHCH}_{2} \mathrm{CH}_{2} \mathrm{OH} \times 2\right), \quad 2.26 \quad$ (brs, 4H, $\left.\mathrm{CH}_{2} \mathrm{NHCH}_{2} \mathrm{CH}_{2} \mathrm{OH} \times 2\right) .{ }^{13} \mathrm{C}-\mathrm{NMR}\left(\mathrm{CDCl}_{3}, 75 \mathrm{MHz}\right) \delta: 148.5$, $143.5,135.9,132.1,131.0,130.4,129.5,128.9,128.6,127.7$, $127.5,122.8,118.2,116.6,61.0,53.3,50.3,37.5$. IR (KBr) $v$ : 2924, 2852, 1597, 1489, 1466, 1400, $1243 \mathrm{~cm}^{-1}$. HR-MS (ESI) Calcd for $\mathrm{C}_{33} \mathrm{H}_{32} \mathrm{ClN}_{2} \mathrm{O}_{3}[\mathrm{M}+\mathrm{H}]^{+}$539.2101, Found 539.2106.

$N^{3}, N^{11}$-Bis(2-hydroxyethyl)-14-(4-bromophenyl)-14Hdibenzo[a,j]xanthene-3,11-dimethanamine (9f): White solid. Yield $82.1 \%$. mp $120-122^{\circ} \mathrm{C} .{ }^{1} \mathrm{H}-\mathrm{NMR}\left(\mathrm{CDCl}_{3}, 300 \mathrm{MHz}\right)$ $\delta: 8.22(\mathrm{~d}, J=8.7 \mathrm{~Hz}, 2 \mathrm{H}, 1,13-\mathrm{H}), 7.72(\mathrm{~d}, J=8.9 \mathrm{~Hz}, 2 \mathrm{H}$, 5, 9-H), 7.69 (d, J=1.1 Hz, 2H, 4, 10-H), 7.52 (dd, $J=8.7$, $1.7 \mathrm{~Hz}, 2 \mathrm{H}, 2,12-\mathrm{H}), 7.44$ (d, J=8.9 Hz, 2H, 6, 8-H), 7.34 (d, $\left.J=8.5 \mathrm{~Hz}, 2 \mathrm{H}, 2^{\prime}, 6^{\prime}-\mathrm{H}\right), 7.20$ (d, J=8.5 Hz, 2H, 3', 5'-H), $6.34(\mathrm{~s}, 1 \mathrm{H}, 14-\mathrm{H}), 3.90\left(\mathrm{~s}, 4 \mathrm{H}, \mathrm{CH}_{2} \mathrm{NHCH}_{2} \mathrm{CH}_{2} \mathrm{OH} \times 2\right)$, $3.66\left(\mathrm{t}, J=5.3 \mathrm{~Hz}, \quad 4 \mathrm{H}, \quad \mathrm{CH}_{2} \mathrm{NHCH}_{2} \mathrm{CH}_{2} \mathrm{OH} \times 2\right), 2.80 \quad(\mathrm{t}$, $\left.J=5.3 \mathrm{~Hz}, \quad 4 \mathrm{H}, \quad \mathrm{CH}_{2} \mathrm{NHCH}_{2} \mathrm{CH}_{2} \mathrm{OH} \times 2\right), \quad 2.24$ (brs, 4H, $\left.\mathrm{CH}_{2} \mathrm{NHCH}_{2} \mathrm{CH}_{2} \mathrm{OH} \times 2\right) .{ }^{13} \mathrm{C}-\mathrm{NMR}\left(\mathrm{CDCl}_{3}, 75 \mathrm{MHz}\right) \delta: 148.5$, $144.0,136.0,131.6,131.0,130.4,129.9,128.9,127.7,127.5$, $122.7,120.2,118.2,116.5,61.0,53.3,50.6,37.6$. IR (KBr) v: 2920, 2848, 1597, 1485, 1466, 1400, $1252 \mathrm{~cm}^{-1}$. HR-MS (ESI) Calcd for $\mathrm{C}_{33} \mathrm{H}_{32} \mathrm{BrN}_{2} \mathrm{O}_{3}[\mathrm{M}+\mathrm{H}]^{+}$583.1596, Found 583.1590.

Cell Culture The antitumor activity testing was performed on 5 human tumor cell lines from three cancer types (leucemia, liver, cervix) using the standard 3-(4,5-dimethylthiazol-2-yl)-2,5-diphenyltetrazolium bromide (MTT)-dye reduction assay.

The tumor cells were cultured in RPMI1640 medium supplemented with $10 \%$ fetal bovine serum and $1 \%$ penicillinstreptomycin under a humidified atmosphere of $5 \% \mathrm{CO}_{2}$ at $37^{\circ} \mathrm{C}$. The density of inoculum depended on the type of tumor cell, and 400-2000 cells were seeded in $100 \mu \mathrm{L}$ of medium per well of 96-well flat-bottomed microplates.

Cell Proliferation The compounds for test were dissolved in dimethylsulfoxide (DMSO) and further diluted in medium, and seven different concentrations were set. The final concentrations of these compounds were $50,25,12.5,6.2,3.1,0.3$, $0 \mu \mathrm{M}$ in medium of 96 -well microplate, respectively, and the volume ratio of DMSO was $0.2 \%$ in medium each well.

After the cells were allowed to adhere for $24 \mathrm{~h}$, the compounds for test were incubated for $48 \mathrm{~h}$ with the tumor cells. The relative amount of live cells was determined using 3-[4,5-dimethylthiazol-2-yl]-2,5-diphenyltetrazolium bromide (a standard colorimetric MTT-test). ${ }^{14)}$ Absorbance was measured at $490 \mathrm{~nm}$ or $570 \mathrm{~nm}$. The drug concentration that caused $50 \%$ cell growth inhibition $\left(\mathrm{IC}_{50}\right)$ was calculated by SPSS program. The results are expressed as mean \pm standard deviation (S.D.) of at least three independent experiments.

Apoptosis Assay HeLa cells were treated with compounds $6 \mathrm{e}$ and $6 \mathrm{f}$ at the concentrations of $12.5 \mu \mathrm{M}$ and $25 \mu \mathrm{M}$ for $24 \mathrm{~h}$, respectively, and then the cells were stained with both Annexin V fluorescein isothiocyanate (FITC) and propidium iodide (PI), and measured by FACSAria flow cytometer (Becton Dickinson, CA, U.S.A.). Flow cytometry data were analyzed by FCS express V3 (De Novo Software, Los Angeles, CA, U.S.A.). 
Acknowledgments The authors thank Dr. Yan Gao and M.E. Xiang Li for testing the ${ }^{1} \mathrm{H}$ - and ${ }^{13} \mathrm{C}-\mathrm{NMR}$ spectra, M.E. Yanchao $\mathrm{Yu}$ and Xuelian Liu for help with the experiment, and Dr. Yi Wang for the checking language.

\section{References}

1) Lambert R. W., Martin J. A., Merrett J. H., Parkes K. E. B., Thomas G. J., PCT Int. Appl. WO 9706178 (1997) [Chem. Abstr., 126, $212377 y$ (1997)].

2) Takeshiba H., Jpn. Tokkyo Koho JP 56005480 (1981) [Chem. Abstr., 95, 80922b (1981)].

3) Poupelin J. P., Saint-Ruf G., Foussard-Blanpin O., Narcisse G., Uchida-Ernouf G., Lacroix R., Eur. J. Med. Chem., 13, 67-71 (1978).

4) Ion R. M., Planner A., Wiktorowicz K., Frackowiak D., Acta Biochim. Pol., 45, 833-845 (1998).

5) Buu-Hoi N. P., Saint-Ruf G., De A., Hieu H. T., Bull. Chim. Ther.,
7, 83-86 (1972).

6) Banerjee A., Mukherjee A. K., Stain Technol., 56, 83-85 (1981).

7) Knight C. G., Stephens T., Biochem. J., 258, 683-687 (1989).

8) Ahmad M., King T. A., Ko D.-K., Cha B. H., Lee J., J. Phys. D Appl. Phys., 35, 1473-1476 (2002).

9) Shenkenberg T. D., Von Hoff D. D., Ann. Intern. Med., 105, 67-81 (1986).

10) Bhattacharya A. K., Rana K. C., Mujahid M., Sehar I., Saxena A. K., Bioorg. Med. Chem. Lett., 19, 5590-5593 (2009).

11) Vilches-Herrera M., Miranda-Sepúlveda J., Rebolledo-Fuentes M., Fierro A., Lühr S., Iturriaga-Vasquez P., Cassels B. K., ReyesParada M., Bioorg. Med. Chem., 17, 2452-2460 (2009).

12) Liang Y. H., He Q. Q., Zeng Z. S., Liu Z. Q., Feng X. Q., Chen F. E., Balzarini J., Pannecouque C., Clercq E. D., Bioorg. Med. Chem., 18, 4601-4605 (2010).

13) Wu D. Q., Pisula W., Haberecht M. C., Feng X. L., Müllen K., Org. Lett., 11, 5686-5689 (2009), Supporting Information, 3.

14) Mosmann T., J. Immunol. Methods, 65, 55-63 (1983). 